\title{
Mitochondrial reprogramming via ATP5H loss promotes multimodal cancer therapy resistance
}

\author{
Kwon-Ho Song, ${ }^{1,2,3}$ Jae-Hoon Kim, ${ }^{4}$ Young-Ho Lee, ${ }^{1,2,3}$ Hyun Cheol Bae, ${ }^{5}$ Hyo-Jung Lee, ${ }^{1,2,3}$ Seon Rang Woo, ${ }^{1,2,3}$ Se Jin Oh, ${ }^{1,2,3}$ \\ Kyung-Mi Lee, ${ }^{1,2}$ Cassian Yee, ${ }^{6}$ Bo Wook Kim, ${ }^{7}$ Hanbyoul Cho, ${ }^{4}$ Eun Joo Chung, ${ }^{8}$ Joon-Yong Chung, ${ }^{9}$ Stephen M. Hewitt, ${ }^{9}$ \\ Tae-Wook Chung, ${ }^{10}$ Ki-Tae Ha, ${ }^{10}$ Young-Ki Bae, ${ }^{11}$ Chih-Ping Mao, ${ }^{12,13,14}$ Andrew Yang, ${ }^{14}$ T.C. Wu, ${ }^{14,15,16,17}$ and Tae Woo Kim ${ }^{1,2,3}$
}

\begin{abstract}
'Department of Biochemistry and Molecular Biology, ${ }^{2}$ Department of Biomedical Science, College of Medicine, and ${ }^{3}$ Translational Research Institute for Incurable Diseases, Korea University College of
Medicine, Seoul, South Korea. ${ }^{4}$ Department of Obstetrics and Gynecology, Gangnam Severance Hospital, Yonsei University College of Medicine, Seoul, South Korea. ${ }^{5}$ Department of Orthopedic Surgery, Seoul National University Hospital, Seoul, South Korea. ${ }^{6}$ Department of Gynecologic Oncology, The University of Texas MD Anderson Cancer Center, Houston, Texas, USA. 'Department of Obstetrics and Gynecology, International St. Mary's Hospital, Catholic Kwandong University College of Medicine, Incheon, Seoul, South Korea. ${ }^{~}$ Radiation Oncology Branch and ${ }^{9}$ Experimental Pathology Laboratory, Laboratory of Pathology, Center for Cancer Research, National Cancer Institute, NIH, Bethesda, Maryland, USA. ${ }^{10}$ Division of Applied Medicine, School of Korean Medicine, Pusan National University, Yangsan, South Korea. ${ }^{11}$ Comparative Biomedicine Research Branch, Research Institute, National Cancer Center, Coyang, South Korea. ${ }^{2} \mathrm{MD}$-PhD Program, ${ }^{13} \mathrm{Immunology}$ Training Program, ${ }^{14} \mathrm{Department} \mathrm{of} \mathrm{Pathology,}{ }^{15} \mathrm{Department}$ of Oncology, ${ }^{16}$ Department of Obstetrics and Gynecology, and ${ }^{17}$ Department of Molecular Microbiology and Immunology, Johns Hopkins School of Medicine, Baltimore, Maryland, USA
\end{abstract}

\begin{abstract}
The host immune system plays a pivotal role in the emergence of tumor cells that are refractory to multiple clinical interventions including immunotherapy, chemotherapy, and radiotherapy. Here, we examined the molecular mechanisms by which the immune system triggers cross-resistance to these interventions. By examining the biological changes in murine and tumor cells subjected to sequential rounds of in vitro or in vivo immune selection via cognate cytotoxic T lymphocytes, we found that multimodality resistance arises through a core metabolic reprogramming pathway instigated by epigenetic loss of the ATP synthase subunit ATP5H, which leads to ROS accumulation and HIF-1 $\alpha$ stabilization under normoxia. Furthermore, this pathway confers to tumor cells a stem-like and invasive phenotype. In vivo delivery of antioxidants reverses these phenotypic changes and resensitizes tumor cells to therapy. ATP5H loss in the tumor is strongly linked to failure of therapy, disease progression, and poor survival in patients with cancer. Collectively, our results reveal a mechanism underlying immune-driven multimodality resistance to cancer therapy and demonstrate that rational targeting of mitochondrial metabolic reprogramming in tumor cells may overcome this resistance. We believe these results hold important implications for the clinical management of cancer.
\end{abstract}

\section{Introduction}

The adaptation of tumor cells to immune surveillance as well as the emergence of refractory tumor cells after initial therapy are responsible for eventual disease relapse, progression, and metastasis. Multiple tumor-intrinsic mechanisms have been proposed to cause tumor drug resistance $(1,2)$, including the overexpression of antiapoptotic factors (3), the ejection of pharmacologic agents from tumor cells via drug transporters (4), the biochemical or structural modification of the drug target (5-8), and the adoption of detoxification or bypass routes (9-14). Furthermore, a recent study provides evidence that preferential selection and subsequent expansion of a subset of stem-like tumor cells with an undifferentiated phenotype contribute to resistance (15). However, the potential link between host immune-intrinsic mechanisms (i.e., cancer immune editing) and the development of resistance to therapy is not well understood. We hypothesized that selection pressures imposed by host immune surveillance

Authorship note: $\mathrm{KHS}, \mathrm{JHK}, \mathrm{YHL}$, and $\mathrm{HCB}$ contributed equally to this work. Conflict of interest: The authors have declared that no conflict of interest exists. Submitted: August 9, 2017; Accepted: June 28, 2018

Reference information: J Clin Invest. 2018;128(9):4098-4114.

https://doi.org/10.1172/JCI96804. may contribute to drug resistance. In this study, we investigated this concept and its underlying mechanisms.

To explore this issue, we developed a system to simulate the evolution of cancer cells in a live host and to dissect the mechanisms responsible for escape from immune defenses mounted by $\mathrm{CD} 8^{+}$cytotoxic $\mathrm{T}$ lymphocytes (CTLs), the predominant arm of the cancer immune surveillance network (16-18). Through this analysis, we report here that loss of the D subunit of mitochondrial ATP synthase (ATP5H) constitutes an important pathway through which cancer evolution occurs in response to immune editing. Mitochondrial ATP5H is a critical component of the energyproducing apparatus in eukaryotic cells; thus, disruptions in ATP synthase expression in cancer cells would be expected to influence tumor metabolism. In fact, altered metabolism is a fundamental feature of cancer and has been shown to contribute to chemo- and radiotherapy resistance (19-21). Tumor mitochondrial dysfunction promotes the development of chemoresistance and has increasingly been recognized as an integral target for new cancer therapy approaches $(22,23)$. Likewise, downregulation of ATP synthase components has been documented in a majority of carcinomas $(24,25)$ and has been associated with chemotherapy resistance (26). However, the underlying mech- 
A

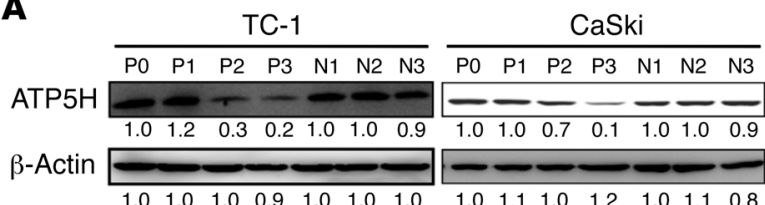

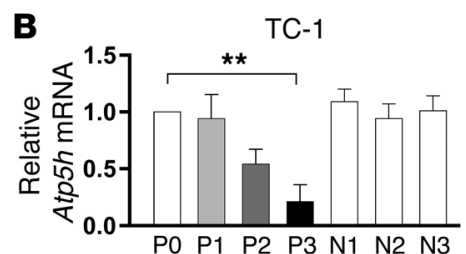

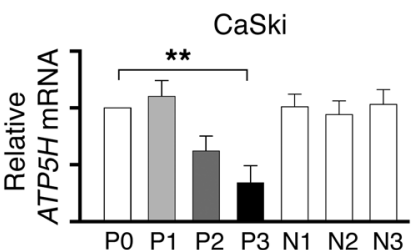

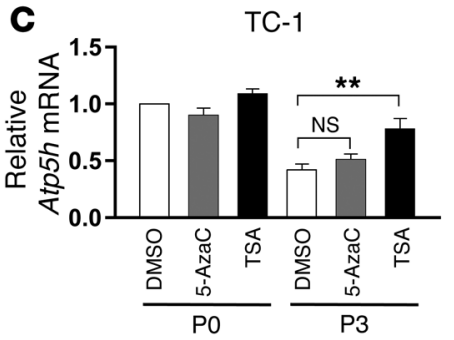

$\mathbf{E}$

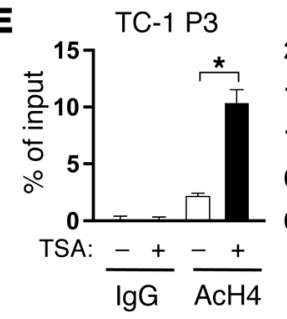

CaSki P3

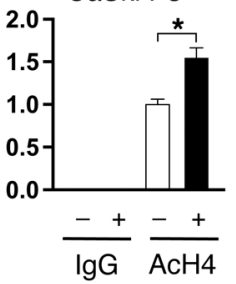

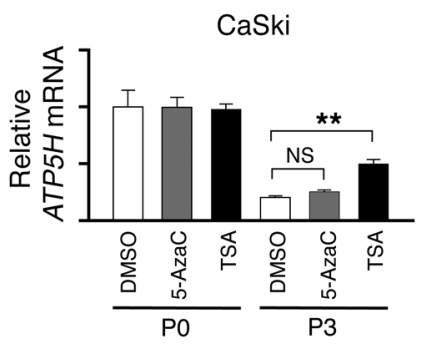

$\mathbf{F}$

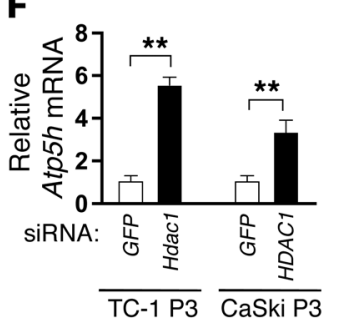

D

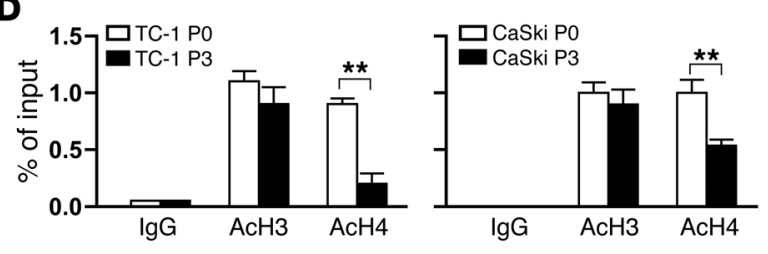

Figure 1. Immune editing triggers epigenetic loss of ATP synthase in tumor cells. (A) ATP5H protein levels in TC-1 or CaSki tumor cells at various stages of immune editing were determined by Western blot analysis (numbers below each blot are densitometric values). (B) mRNA levels of Atp5h (TC-1 cells) or ATP5H (CaSki cells) in TC-1 or CaSki tumor cells at various stages of immune editing were determined by qRT-PCR. (A and B) N1, N2, and N3 were generated through serial selection by irrelevant antigen specific CTLs and were used as negative controls. (C) TC-1 or CaSki P0 and P3 tumor cells were treated with DMSO, 5-AzaC $(5 \mu \mathrm{M})$, or TSA (100 nM). Atp5h (TC-1 cells) or ATP5H (CaSki cells) mRNA levels in these cells were probed by qRT-PCR. (D) The histone acetylation status of TC-1 or CaSki PO and P3 cells was determined by ChIP using anti-histone $\mathrm{H} 3$ and $\mathrm{H} 4$ antibodies, followed by qPCR for the Atp5h or ATP5H promoter locus. (E) TC-1 or CaSki P3 cells were treated with or without TSA, and histone H4 acetylation at the Atp5h or ATP5H promoter was determined by ChIP-qPCR. (F-H) TC-1 or CaSki P3 cells were transfected with the indicated siRNAs. (F) Atp5h or ATP5H mRNA levels were determined by qPCR. (C) Protein levels of HDAC1 and ATP5H were determined by Western blot analysis (numbers below each blot are densitometric values). (H) Relative levels of $\mathrm{AcH} 4$ at the Atp5h or ATP5H promoter were assessed by qChIP. All experiments were performed in triplicate. ${ }^{*} P<0.05$ and ${ }^{*} P<0.01$, by 1-way ANOVA (B and $\mathbf{C}$ ) or 2-tailed Student's $t$ test $(\mathbf{D}-\mathbf{F}$ and $\mathbf{H})$. Data represent the mean \pm SD.

anisms responsible for linking ATP synthase loss to drug resistance have remained unclear.

In this study, we focused on: (a) the mechanisms by which immune editing leads to silencing of ATP5H, and (b) the downstream pathways by which ATP5H loss promotes multimodal resistance to therapy. We discovered that immune editing alters the epigenetic landscape of tumor cells via histone deacetylase 1 (HDAC1) upregulation (27), which turns off ATP5H expression via promoter deacetylation. The epigenetic loss of ATP5H facilitates cancer immune escape and multimodal resistance to immunotherapy, chemotherapy, and radiotherapy. Mechanistically, ATP5H lossinduced mitochondrial reprogramming leads to the accumulation of ROS within tumor cells, which stabilizes HIF-1 $\alpha$ under normoxia and activates the AKT/ERK signaling pathway. Rational targeting of this pathway via antioxidants reverses resistance to therapy. In the clinical setting, we used tumor tissue biopsies from cancer patients and found a strong correlation between loss of ATP synthase and overall survival. Our results demonstrate that epigenetic-mediated mitochondrial metabolic reprogramming in the course of immune editing promotes cancer immune escape and therapy resistance. This pathway provides a target for clinical intervention.

\section{Results}

Immune editing triggers multimodal resistance to chemotherapy and radiotherapy. We first asked whether tumor cells acquire multimodal resistance to therapy through immune editing. For this, we subjected mouse or human tumor cells transformed with HPV oncoproteins (TC-1 or CaSki, respectively) to 3 rounds of in vivo or in vitro selection by cognate CTLs, respectively, as described previously $(18,28)$. At the end of the selection process, the tumor cells (termed P3) were refractory to apoptotic death by cognate CTLs, whereas the parental cells (termed PO), or tumor cells mixed for 3 rounds with noncognate T cells, remained sensitive to cognate CTLs $(18,28)$. We compared the susceptibility of P3 versus PO cells to various classes of chemotherapy or radiotherapy. On the basis of the $\mathrm{LD}_{50}$ values, we found that $\mathrm{P} 3$ cells were 10-fold less sensitive to the pharmacologic agents cisplatin or 5-fluorouracil (5-FU) relative to PO cells (Supplemental Figure 1, A and B; supplemental material available online with this article; https://doi.org/10.1172/JCI96804DS1). P3 cells were also much less sensitive to irradiation-induced apoptosis compared with PO cells (Supplemental Figure 1C). Our results indicate that immune editing of tumor cells can provoke multimodal resistance to chemotherapy, radiotherapy, as well as immunotherapy. 
A

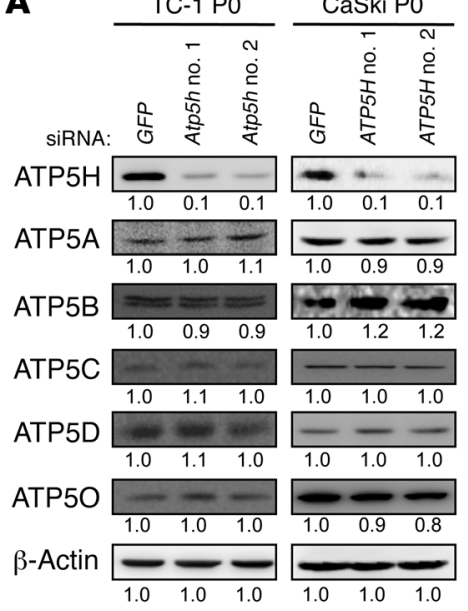

E

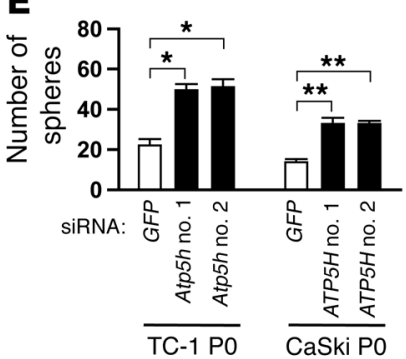

B

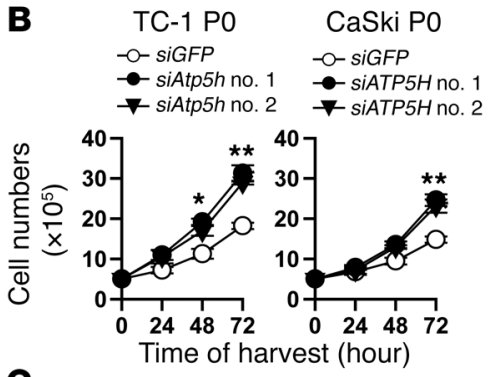

C

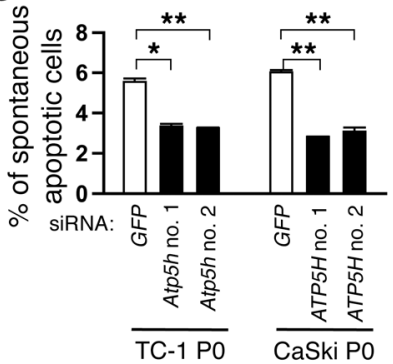

F

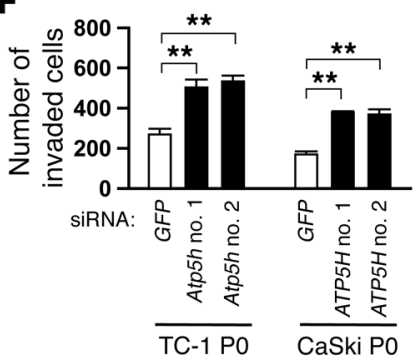

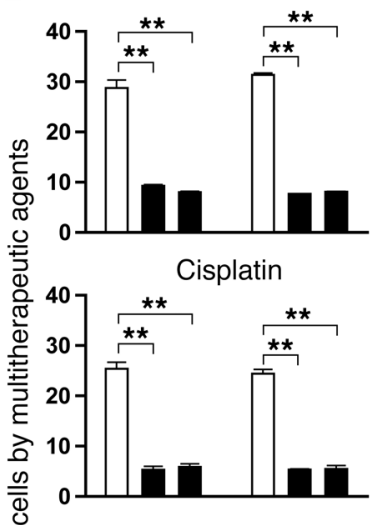

$\gamma$-Irradiation

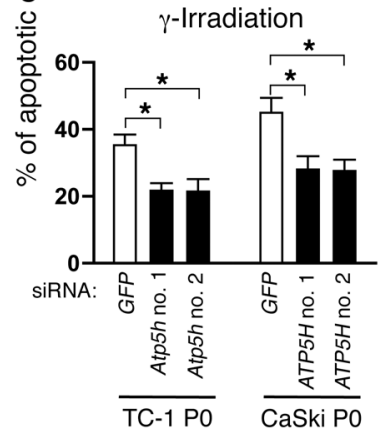

Figure 2. Loss of ATP5H converts tumor cells to resistant, stem-like, and invasive phenotypes. (A-F) TC-1 or CaSki PO cells were transfected with the indicated siRNAs. (A) Levels of the various individual protein components of the ATP synthase complex in these cells were probed by Western blotting (numbers below the blots are densitometric values). (B) Cells were harvested at the indicated time points and counted after trypan blue staining to exclude dead cells. (C) After 72 hours, spontaneous apoptotic cells were determined by flow cytometric analysis of caspase-3 activation. (D) Cells were exposed to tumor-specific CTLs, cisplatin, or radiation, and the frequency of apoptotic cells was determined by flow cytometric analysis of caspase-3 activation. (E) The degree of stem-like and (F) invasive phenotypes in these cells was determined by sphere-forming or Matrigel migration assay. All experiments were performed in triplicate. ${ }^{*} P<0.05$ and ${ }^{* *} P<0.01$, by 2 -way ANOVA (B) or 1 -way ANOVA (C-F). Data represent the mean \pm SD.

Immune editing triggers epigenetic silencing of ATP synthase. To elucidate the underlying mechanisms responsible for multimodal resistance during selection by immune editing, we performed 2D protein electrophoresis and mass spectrometry in lysates derived from P0 or P3 cells. From this analysis, we noted that subunit D of the ATP synthase complex (ATP5H) was downregulated in P3 cells compared with that detected in PO cells (Supplemental Figure 2A and Supplemental Table 1; this was then confirmed by Western blot analysis (Supplemental Figure 2B). We observed a gradual loss of the ATP5H protein in both murine and human tumor cells over the course of immune editing (Figure 1A). This gradual protein loss was accompanied by decreased Atp5h and ATP5H mRNA expression, indicating that ATP5H loss was occurring at the transcriptional level (Figure 1B). We reasoned that epigenetic changes in tumor cells in the course of immune editing mediate transcriptional loss of ATP5H. To test this, we treated tumor cells (TC-1 or CaSki), before (P0) or after (P3) sequential immune editing, with the DNA methyltransferase (DNMT) inhibitor 5-azacytidine (5-AzaC) or the HDAC inhibitor trichostatin A (TSA). Exposure to TSA, but not 5-AzaC, upregulated Atp5h and ATP5H transcription in P3 TC-1 and CaSki cells, respectively (Figure 1C). This suggests that immune editing may induce epigenetic silencing of ATP5H via histone deacetylation. To verify this, we performed ChIP, pulling down histone $\mathrm{H} 3$ and $\mathrm{H} 4$ and then probing for associated Atp5h or ATP5H promoter sequences. We found that histone $\mathrm{H} 4$ acetylation (AcH4) at the Atp5h and $A T P 5 H$ promoter regions was indeed reduced in P3 cells relative to P0 cells (Figure 1D), implying immune editing-mediated deacetylation of histone $\mathrm{H} 4$. Notably, histone $\mathrm{H} 4$ deacetylation at the Atp5h and ATP5H promoter loci in tumor cells after selection was reversed by exposure to TSA (Figure 1E). Consistent with the unresponsive effects of 5-AzaC on Atp5h expression, we did not detect differences in DNA methylation between tumor cells before or after immune editing (Supplemental Figure 3), which suggests that histone deacetylation is the primary mechanism underlying ATP5H epigenetic silencing.

We next investigated the factor mediating histone deacetylation at the Atp5h and ATP5H loci during immune editing. Recently, we discovered that upregulation of HDAC1 is important for epigenetic reprogramming and acquisition of both stem-like properties and resistance to CTLs by CaSki P3 cells (27). Consistently, we observed HDAC1 upregulation upon in vivo immune editing of TC-1 tumor cells (Supplemental Figure 4). We hypothesized that 
A
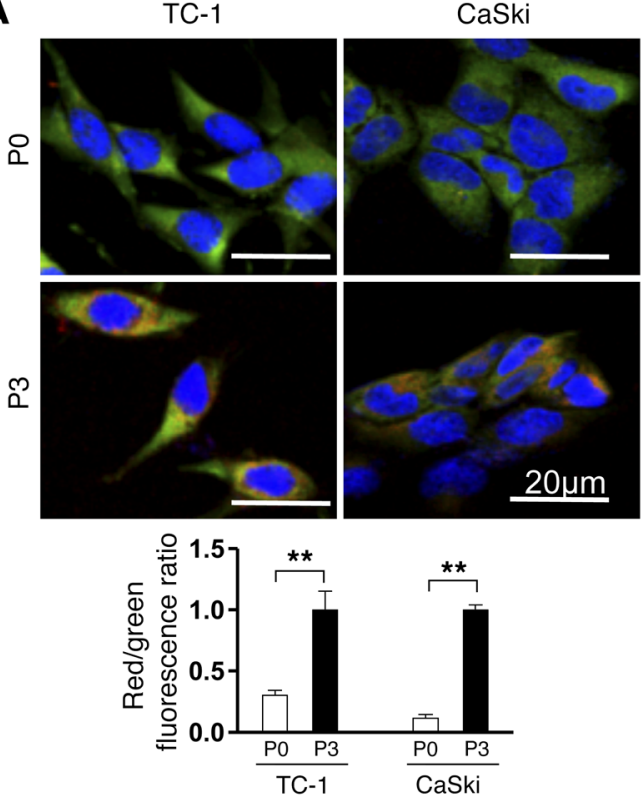

B

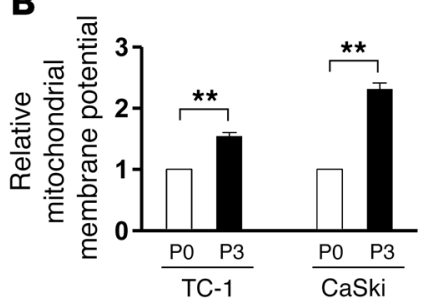

D

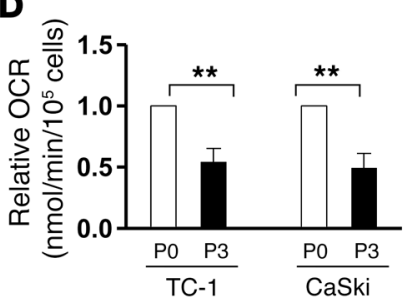

C

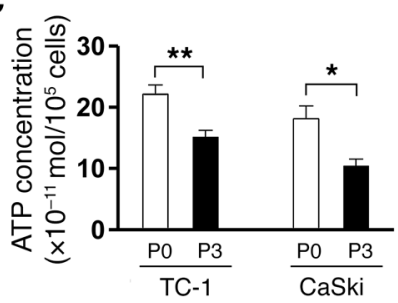

E

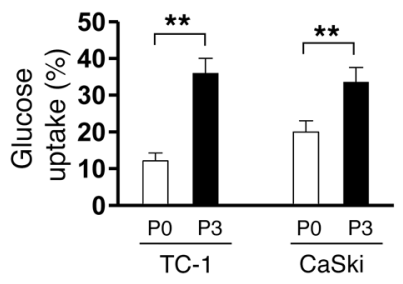

$\mathbf{F}$

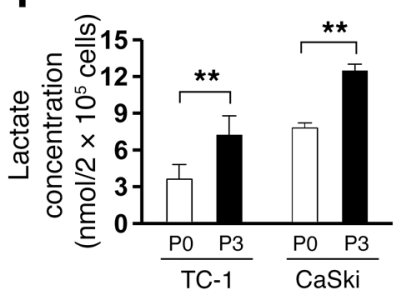

G

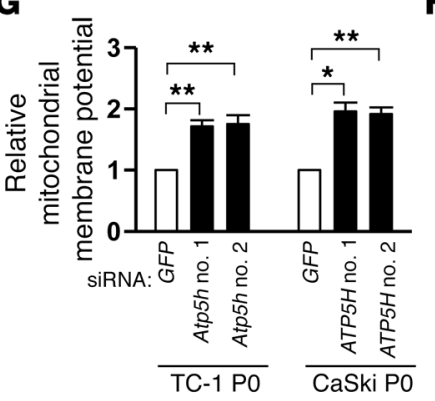

H

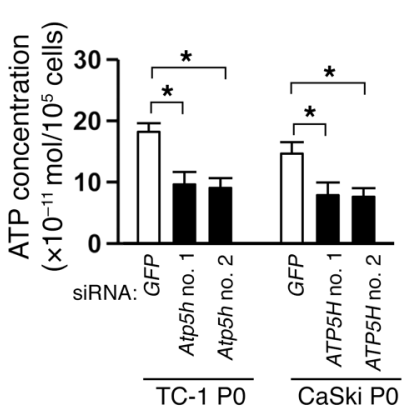

I

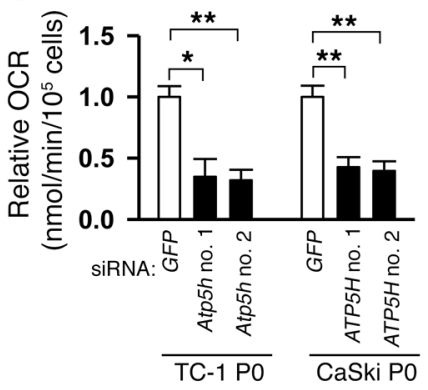

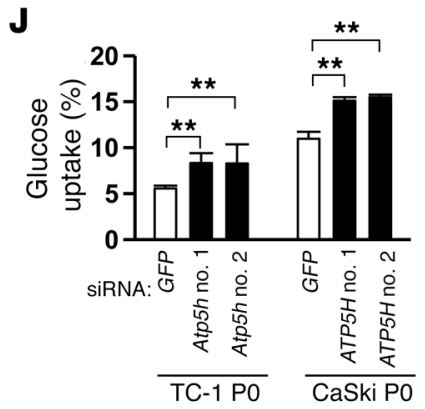

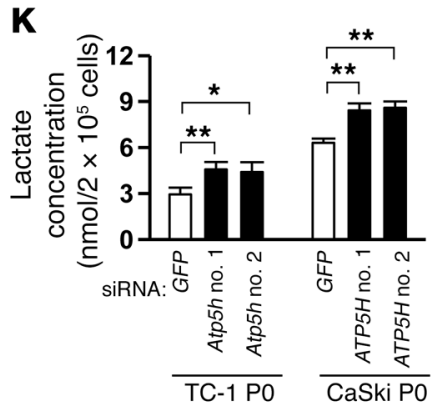

Figure 3. Immune editing-induced ATP5H loss triggers mitochondrial metabolic reprogramming. (A) Mitochondrial membrane potential was measured with JC-1 staining. TC-1 or CaSki cells without (PO) or with (P3) immune editing were stained with JC-1 and then visualized by laser-scanning confocal microscopy. Images are representative of 3 separate experiments. Scale bars: $20 \mu \mathrm{m}$. Graph shows the ratio of red to green fluorescence intensity. (B) Relative mitochondrial membrane potential in P0 versus P3 cells was quantified in JC-1-stained cells by flow cytometry. (C) ATP concentration in PO and P3 cells was measured by luciferase-based ATP assay. (D) Relative OCR in PO versus P3 cells was measured with an extracellular flux analyzer. (E) PO and P3 cells were incubated with 2-NBDG for 20 minutes. Intracellular fluorescence of 2-NBDG was detected by flow cytometry. (F) Intracellular lactate levels in PO and P3 cells were measured by lactate colorimetric assay. (G-K) TC-1 or CaSki PO cells were transfected with the indicated siRNAs. (G) Relative mitochondrial membrane potential was determined by JC-1 staining. (H) ATP concentration in these cells was measured by luciferase-based ATP assay. (I) Relative OCR was measured with an extracellular flux analyzer. (J) Cells were incubated with 2-NBDG for 20 minutes. The intracellular fluorescence of 2-NBDG was detected by flow cytometry. (K) Lactate levels in these cells were measured by lactate colorimetric assay. All experiments were performed in triplicate. ${ }^{*} P<0.05$ and ${ }^{* *} P<0.01$, by 2 -tailed Student's $t$ test $(\mathbf{A}-\mathbf{F})$ or 1 -way ANOVA (G-K). Data represent the mean \pm SD.

HDAC1 might be responsible for transcriptional loss of ATP5H. Indeed, knockdown of Hdac1 or HDAC1 with siRNA in P3 cells raised mRNA and protein levels of ATP5H, which was accompanied by increased histone $\mathrm{H} 4$ acetylation at the Atp5h and $A T P 5 H$ promoters (Figure 1, F-H). Altogether, our data demonstrate that immune editing facilitates epigenetic silencing of ATP5H via HDAC1-mediated histone deacetylation.

ATP5H loss confers a stem-like, invasive phenotype to tumor cells as well as multimodal resistance to immunotherapy, chemotherapy, and radiotherapy. Previously, we found that immune-edited P3 
A

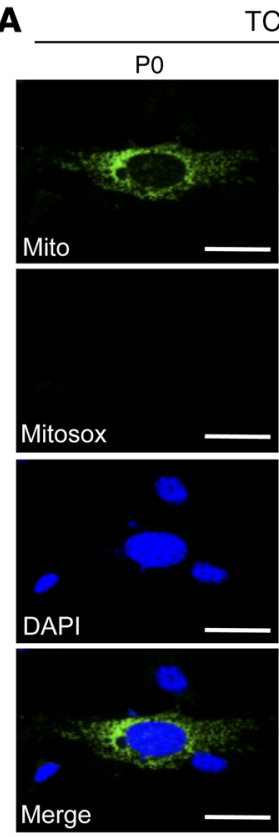
TC-

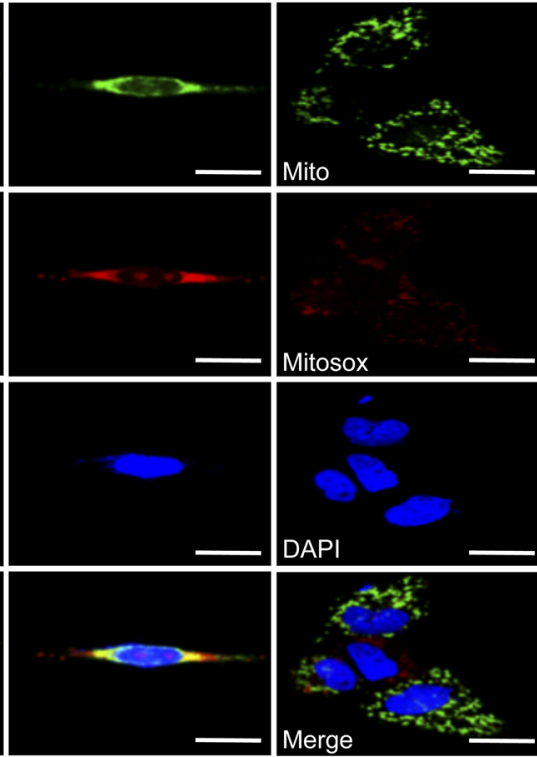

CaSki

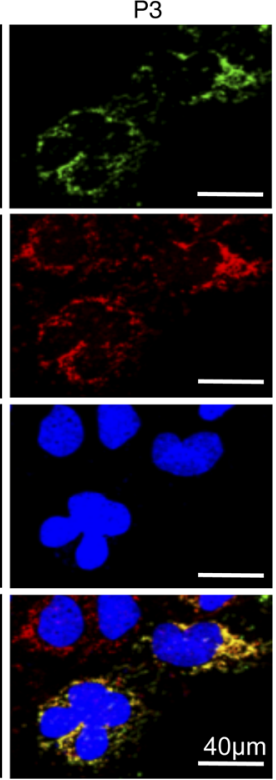

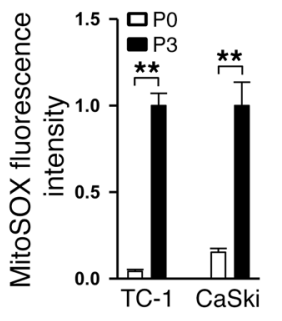

B
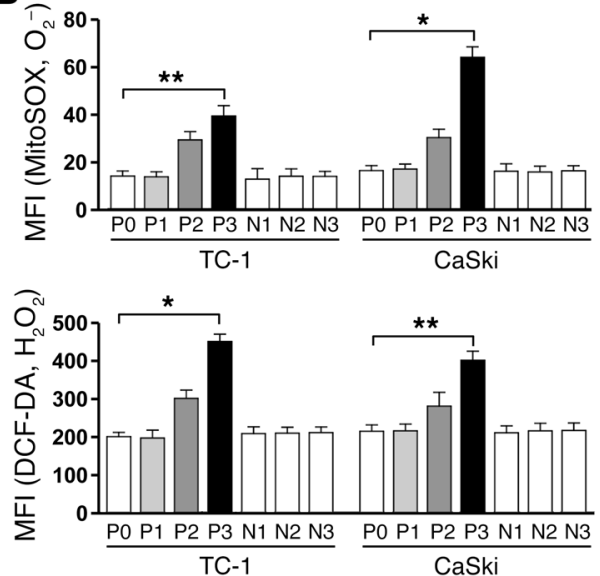

C

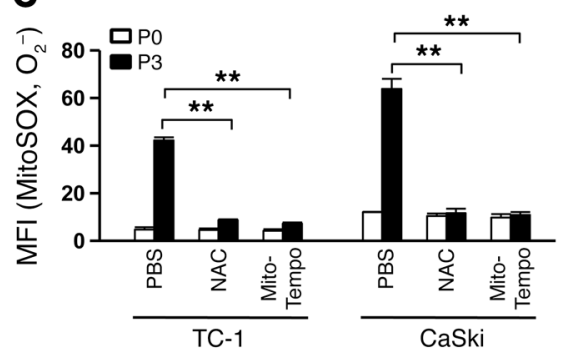

D

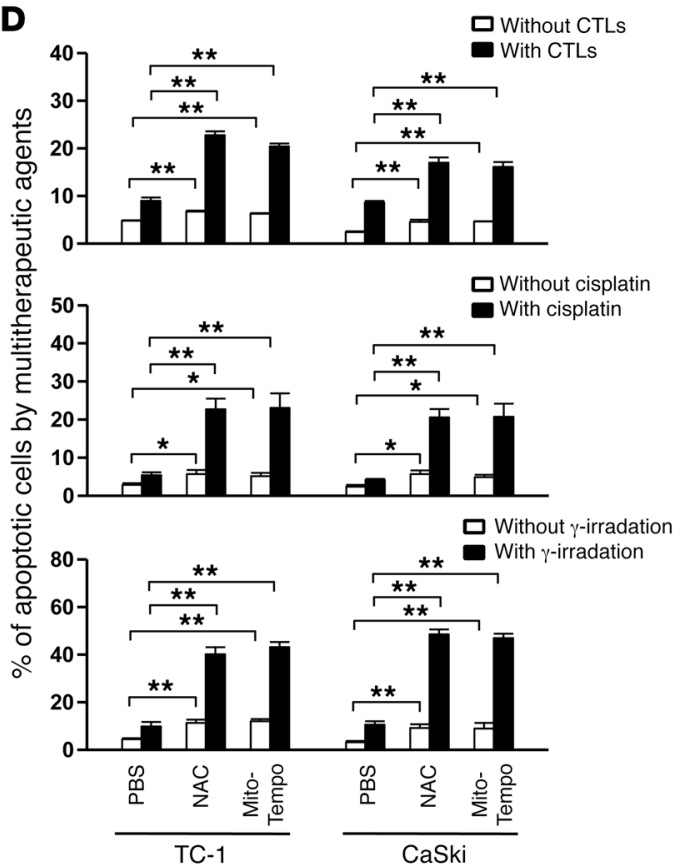

E
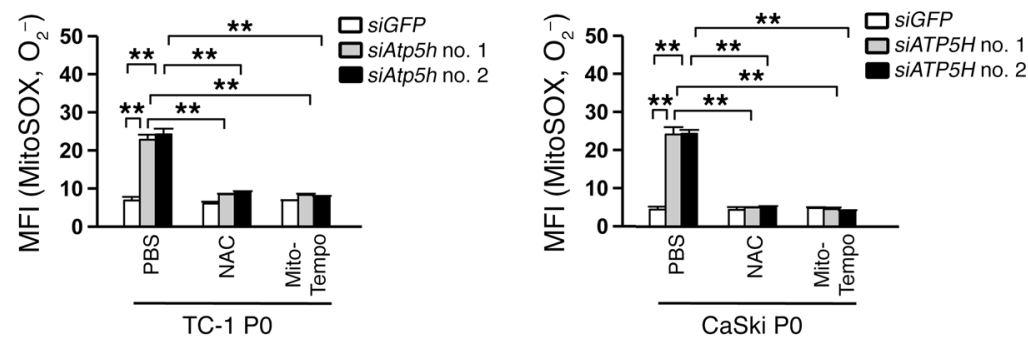

$\mathbf{F}$

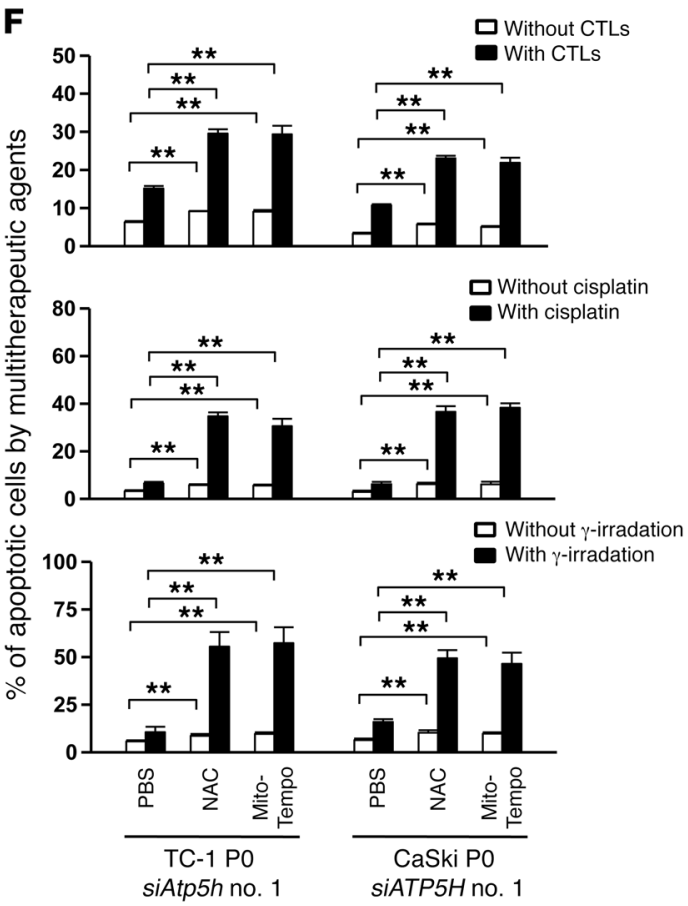


Figure 4. ATP5H loss-induced ROS accumulation converts tumor cells to resistant phenotypes after immune editing. (A) PO or P3 TC-1 or CaSki cells were stained for total mitochondria (Mito, green), mitochondrial superoxide (MitoSOX, red), and nuclei (DAPI, blue). Cells were visualized under a laser-scanning confocal microscope. Graph depicts the experimental quantitation of MitoSOX fluorescence intensity. Scale bars: 40 $\mu \mathrm{m}$. (B) The abundance of ROS $\left(\mathrm{O}_{2}{ }^{-}, \mathrm{H}_{2} \mathrm{O}_{2}\right)$ in TC-1 or CaSki tumor cells at various stages of immune editing was determined by MitoSOX and DCFHDA staining, followed by flow cytometric analysis. Data are presented as the mean fluorescence intensity (MFI). (C) P0 or P3 cells were treated with PBS, NAC, or MitoTempo. The abundance of ROS in these cells was determined by MitoSOX staining, followed by flow cytometric analysis. (D) TC-1 or CaSki P3 cells were treated with PBS, NAC, or MitoTempo and then exposed to tumor-specific CTLs, cisplatin, or $\gamma$-irradiation. The percentage of apoptotic cells was measured by flow cytometric analysis of caspase-3 activation. (E) TC-1 or CaSki PO cells were transfected with the indicated siRNAs. Cells were treated with PBS, NAC, or MitoTempo. The abundance of ROS in these cells was determined by MitoSOX staining, followed by flow cytometric analysis. (F) siAtp5h no. 1-transfected TC-1 or siATP5H no. 1-transfected CaSki PO cells were treated with PBS, NAC, or MitoTempo. Cells were exposed to E7-specific CTLs, cisplatin, or $\gamma$-irradiation. The frequency of apoptotic cells was determined by flow cytometric analysis of caspase-3 activation. All experiments were performed in triplicate. ${ }^{*} P<0.05$ and ${ }^{*} P<0.01$, by 2 -tailed Student's $t$ test (A), 1-way ANOVA (B), or 2-way ANOVA (C-F). Data represent the mean \pm SD.

tumor cells have more tumorigenic, stem-like, and invasive properties relative to parental PO tumor cells $(16,29)$. To determine the effects of ATP5H loss on the phenotype of tumor cells, we silenced Atp5h or ATP5H expression in PO cells using siRNA (Figure 2A). Compared with siGFP-transfected control cells, siAtp5h or siATP5H transfection increased the number of viable PO cells over time and reduced spontaneous apoptosis of transfected PO cells (Figure 2, B and C). Furthermore, siAtp5h or siATP5H transfection in PO tumor cells induced multimodal resistance to immunotherapy, chemotherapy, and radiotherapy (Figure 2D) and conferred a stem-like, invasive phenotype (Figure 2, E and F, and Supplemental Figure 5). Our data indicate that loss of ATP5H is capable of phenocopying P3 by converting the tumor cells to antiapoptotic, treatment-resistant, stem-like, and invasive phenotypes.

ATP5H loss following immune editing triggers mitochondrial metabolic reprogramming. Because ATP5H is an integral part of the ATP synthase apparatus, we postulated that immune editing would also cause mitochondrial dysfunction and metabolic reprogramming. Indeed, relative to PO cells, P3 cells exhibited elevated mitochondrial membrane potential (Figure 3, A and B), defective ATP production (Figure 3C), reduced oxygen consumption (Figure 3D), enhanced glucose uptake (Figure 3E), and increased lactate production (Figure 3F). Knockdown of Atp5h or ATP5H in PO cells with siRNA phenocopied these events (Figure $3, \mathrm{G}-\mathrm{K})$. Interestingly, there was no alteration in AMPK signaling or induction of spontaneous autophagy in P3 cells or siAtp5h-or siATP5H-transfected PO cells compared with control and siGFPtransfected PO cells, suggesting that loss of ATP5H does not exert significant metabolic stress on tumor cells (Supplemental Figure 6). Therefore, we conclude that ATP synthase, and in particular the ATP5H subunit, is downregulated as tumor cells undergo immune editing. In turn, this loss of ATP synthase triggers mitochondrial metabolic reprogramming.
ATP5H loss-induced ROS accumulation mediates resistance to cancer therapy. It has been documented that increased mitochondrial membrane potential leads to ROS production $(30,31)$. In addition, mitochondrial dysfunction-induced production of ROS was reported to affect the chemoresistance phenotypes and malignant behavior of cancer cells (32). In this regard, we observed high levels of ROS in P3 cells compared with levels in PO cells (Figure $4 \mathrm{~A}$ ), and ROS levels increased gradually with sequential rounds of immune editing from PO to P3 cells (Figure 4B). To directly link ROS accumulation to phenotypes of P3 tumor cells, we exposed the P3 tumor cells to various therapeutic methods, with or without supplementation of antioxidants ( $N$-acetyl cysteine [NAC]) or a mitochondria-specific ROS scavenger (MitoTempo). The presence of NAC or MitoTempo drastically reduced ROS levels in P3 cells (Figure 4C) and restored the susceptibility of P3 cells to immunotherapy, chemotherapy, and radiotherapy (Figure 4D), suggesting a crucial role of ROS in multimodal resistance of immune-edited cancer cells. We hypothesized that the refractory phenotype of tumor cells with deficient ATP5H was due to ROS accumulation elicited by mitochondrial dysfunction. Notably, knockdown of Atp5h or ATP5H in PO cells increased ROS levels that could subsequently be reversed by NAC or MitoTempo supplementation (Figure 4E). Likewise, NAC or MitoTempo treatment increased the sensitivity of siAtp5h- and siATP5H-transfected PO cells to immunotherapy, chemotherapy, and radiotherapy (Figure 4F). Of note, we observed no elevation of endogenous antioxidant enzymes in P3 cells or siATP5H-transfected PO cells compared with control PO cells (Supplemental Figure 7, A and B). Furthermore, the increased ROS levels in P3 cells and siAtp5h- and siATP5H-transfected PO cells compared with control PO cells did not increase the amount of DNA damage observed in the cells (Supplemental Figure 7, C and D). This further suggests that the ROS induced by ATP5H loss does not cause significant oxidative toxicity to TC-1 or CaSki tumor cells.

Since we observed that knocking down ATP5H in PO cells phenocopied various properties of the $\mathrm{P} 3$ cells, we reasoned that restoring ATP5H expression in $\mathrm{P} 3$ cells could similarly reverse the properties of P3 cells to resemble those of PO cells. Indeed, transfection of CaSki P3 cells with ATP5H (Figure 5A) reduced the mitochondrial membrane potential (Figure $5 \mathrm{~B}$ ), increased ATP production and oxygen consumption (Figure 5, C and D), and reduced glucose uptake and lactate production (Figure 5, E and F). Furthermore, restoration of ATP5H expression in P3 cells reduced ROS levels (Figure 5G) and resensitized P3 cells to immunotherapy, chemotherapy, and radiotherapy (Figure 5, H-J). Given these data, we conclude that ATP5H loss-induced ROS accumulation mediates broad-spectrum cross-resistance to cancer therapy.

Delivery of antioxidants overcomes immune and drug resistance in vivo. Given our observations in vitro, we reasoned that in vivo infusion of antioxidants should reverse multimodal resistance to therapy. To test this, we treated TC-1 P3-bearing C57BL/6 mice with immunotherapy along with s.c. injection of hydrogel-encapsulated NAC (Figure 6A). While immunotherapy alone had no effect on tumor growth, dual therapy with E7-specific CTLs and NAC retarded tumor growth (Figure 6, B and C) and prolonged survival of the mice (Figure 6D). Likewise, in CaSki P3-bearing NOD/SCID mice (Figure 6E), while cisplatin monotherapy was 

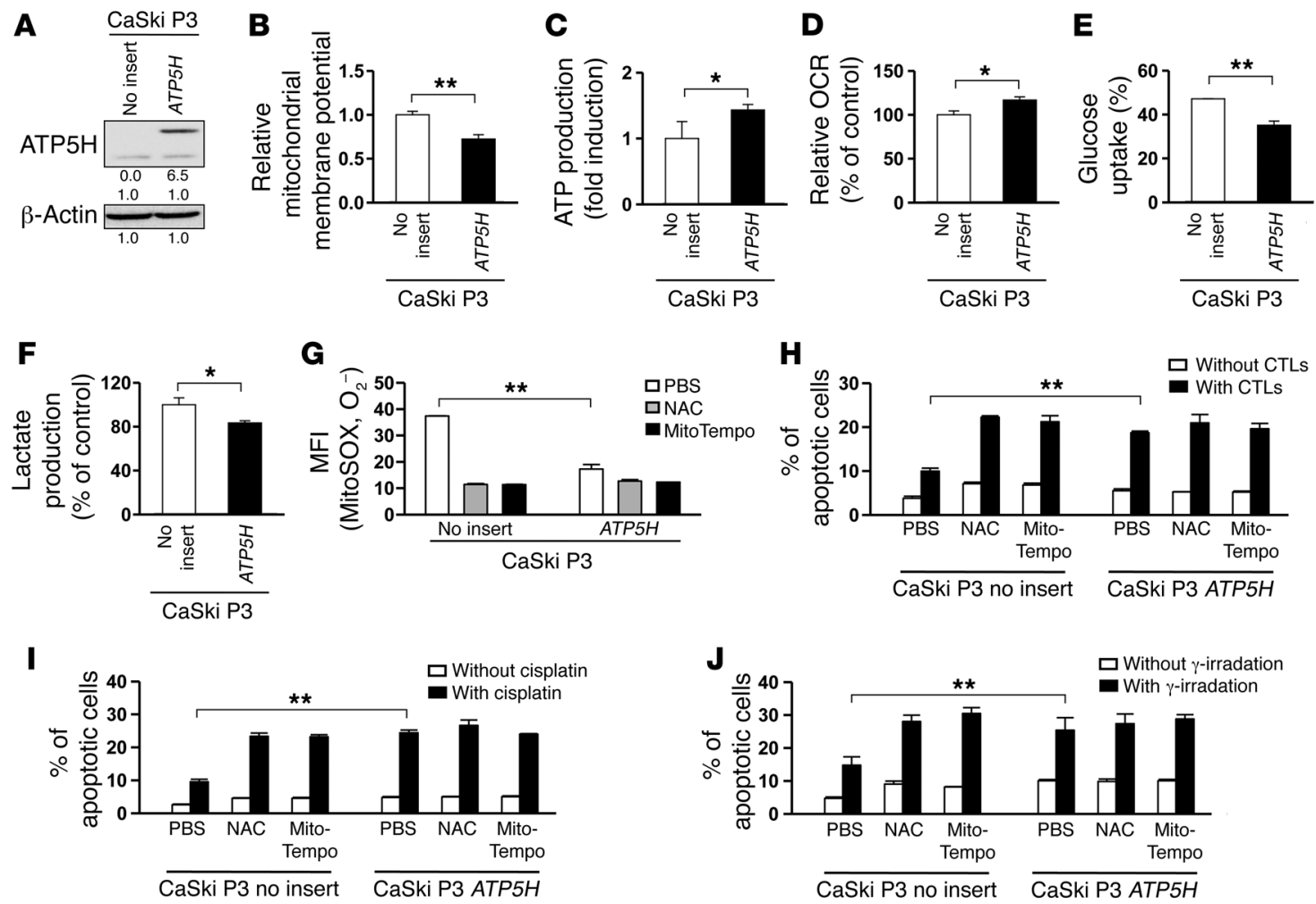

Figure 5. Restoration of ATP5H reverses mitochondrial metabolism and resistant phenotypes of P3 tumor cells. (A-F) CaSki P3 cells were transfected with empty vector (No insert) or ATP5H-FLAG. (A) Levels of endogenous (lower band) and exogenous ATP5H protein (upper band) in these cells were analyzed by immunoblotting. Numbers below the blots indicate the fold value for endogenous protein levels of P3 no-insert cells. (B) Relative mitochondrial membrane potential in P3 no-insert versus P3 ATP5H cells was quantified in JC-1-stained cells by flow cytometric analysis. (C) ATP concentration in P3 no-insert and P3 ATP5H cells was measured by luciferase-based ATP assay. (D) Relative OCR in P3 no-insert versus P3 ATP5H cells was measured with an extracellular flux analyzer. (E) P3 no-insert and P3 ATP5H cells were incubated with 2-NBDC for 20 minutes, followed by intracellular fluorescence of 2-NBDC detection by flow cytometric analysis. (F) Intracellular lactate levels in P3 no-insert and P3 ATP5H cells were measured by lactate colorimetric assay. (C) P3 no-insert and P3 ATP5H cells were treated with PBS, NAC, or MitoTempo. The abundance of ROS in these cells was determined by MitoSOX staining, followed by flow cytometric analysis. (H-J) P3 no-insert and P3 ATP5H cells were treated with PBS, NAC, or MitoTempo, and then cells were exposed to tumor-specific CTLS (H), cisplatin (I), or $\gamma$-irradiation (J). The percentage of apoptotic cells was measured by flow cytometric analysis of caspase-3 activation. All experiments were performed in triplicate. ${ }^{*} P<0.05$ and ${ }^{* *} P<0.01$, by 2-tailed Student's $t$ test (B-F), 1-way ANOVA (G), or 2 -way ANOVA (H-J). Data represent the mean \pm SD.

futile, cisplatin combined with NAC curbed tumor growth (Figure 6, F and $\mathrm{G}$ ) and prolonged survival (Figure $6 \mathrm{H}$ ). Thus, we conclude that delivery of antioxidants can overcome multimodality resistance of immune-edited tumor cells to therapy.

Loss of ATP synthase activates the HIF-1 pathway through ROS-mediated normoxic HIF-1 1 stabilization. We next examined the molecular pathways downstream of mitochondrial metabolic reprogramming and ROS accumulation that promote multimodal resistance to therapy as a result of ATP5H loss. In this regard, we have previously shown that normoxic HIF-1 $\alpha$ is markedly upregulated in tumor cells following selection by immune editing (28). While we have established that HIF-1 $\alpha$ drives multimodality resistance (Supplemental Figure 8), it is unclear how HIF-1 $\alpha$ protein expression is induced in immune-edited tumor cells under normoxic conditions. Notably, HIF-1 $\alpha$ protein levels before and after immune editing are regulated primarily at the translational level rather than the transcriptional level, as evidenced by identical mRNA levels in PO and P3 cells (Figure 7A). Under normal oxygen tension, HIF-1 $1 \alpha$ becomes hydroxylated and is subsequently targeted for degradation by the E3 ubiquitin ligase Von Hippel-Lindau
(VHL) (33-35). We therefore measured hydroxylated HIF-1 $\alpha$ and VHL protein levels in $\mathrm{PO}$ versus $\mathrm{P} 3$ cells. While $\mathrm{PO}$ and $\mathrm{P} 3$ cells had similar levels of VHL, the level of hydroxylated HIF-1 $\alpha$ was much lower in P3 cells than in P0 cells (Figure 7B). The presence of ROS has been shown to inhibit conversion of HIF-1 $\alpha$ to the hydroxylated state $(36,37)$. Indeed, infusion of antioxidants produced hydroxylated HIF-1 $\alpha$ and markedly reduced HIF-1 $\alpha$ levels (Figure 7C). This was accompanied by a loss of signaling of other oncogenic components, AKT/ERK and VEGF (Figure 7, C and D). Antioxidant infusion did not further reduce the level of AKT/ERK signaling in P3 cells transfected with siHifla or siHIF1A (Supplemental Figure 9), suggesting that ROS mediate AKT/ERK signaling through the expression of HIF-1, as reported previously (28).

We then investigated the relationship between ATP5H status and HIF-1 $\alpha$ expression. Compared with control siGFP-transfected cells, siAtp5h-and siATP5H-transfected PO cells had 4- to 10-fold overexpression of HIF-1 $\alpha$ (Figure 7E). Furthermore, we found that siAtp5h- and siATP5H-transfected PO cells had markedly higher levels of AKT/ERK phosphorylation (p-AKT/p-ERK) and VEGF secretion (Figure 7, E and F). In line with this observation, resto- 

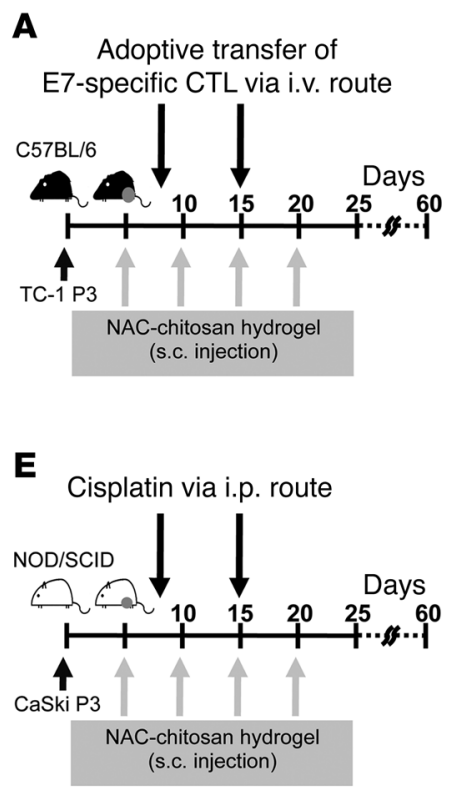

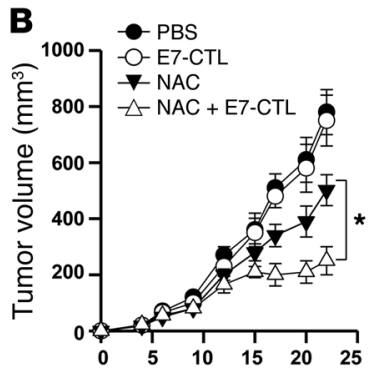

Days after tumor challenge

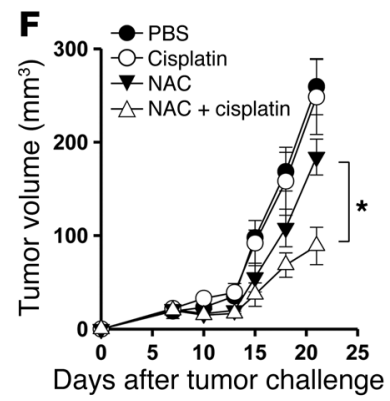

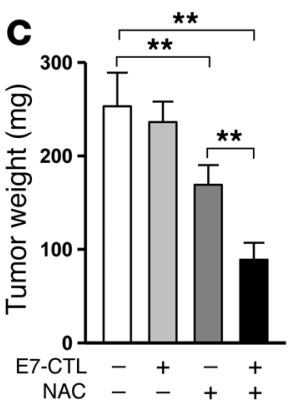
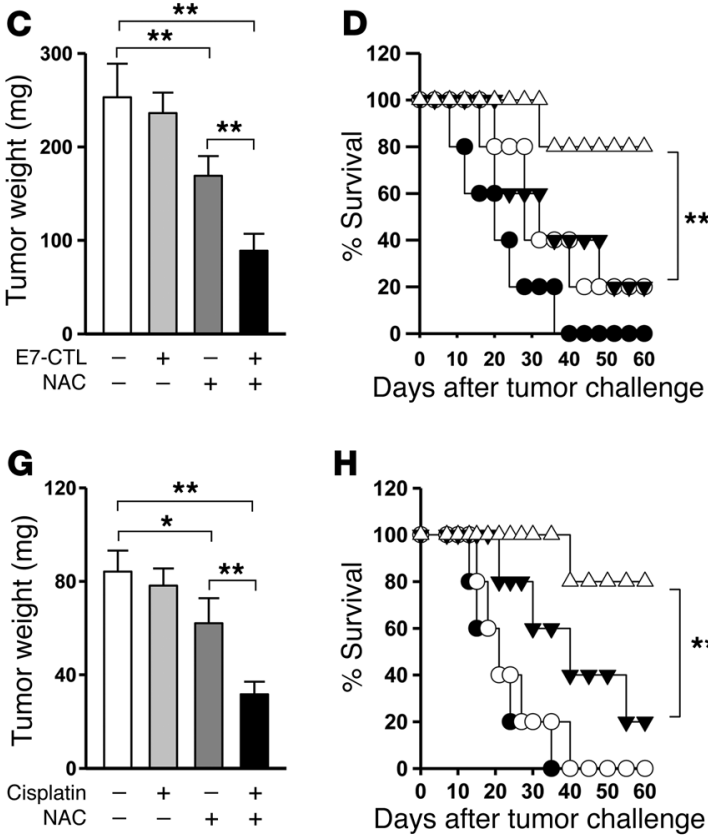

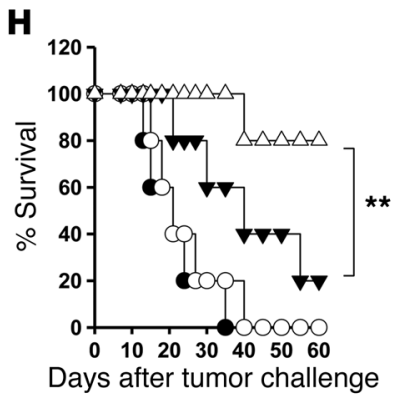

Figure 6. Delivery of antioxidants reverses resistance-to-therapy phenotype after immune editing. (A) $\mathrm{C} 5 \mathrm{BL} / 6$ mice were inoculated with refractory TC-1 cells (P3). Mice were administered antioxidants ( $0.1 \mathrm{mg} / \mathrm{kg} \mathrm{NAC)} \mathrm{via} \mathrm{chitosan} \mathrm{hydrogel,} \mathrm{together} \mathrm{with} \mathrm{adoptive} \mathrm{transfer} \mathrm{of} \mathrm{E7-specific} \mathrm{CTLs} \mathrm{at} \mathrm{the}$ indicated time points. (B) Tumor size was measured every 3 days. (C) The average tumor weight in each group was measured 21 days after tumor challenge. (D) Kaplan-Meier analysis of survival in each group. (E) NOD/SCID mice were inoculated with refractory CaSki cells (P3). Mice were administered $0.1 \mathrm{mg} /$ $\mathrm{kg} \mathrm{NAC} \mathrm{via} \mathrm{chitosan} \mathrm{hydrogel,} \mathrm{together} \mathrm{with} 2 \mathrm{mg} / \mathrm{kg}$ cisplatin at the indicated time points. (F) Tumor size was measured every 3 days. (G) The average tumor weight in each group was measured 21 days after tumor challenge. (H) Kaplan-Meier analysis of survival in each group. In vitro experiments were performed in triplicate. For in vivo experiments, 10 mice from each group were used. ${ }^{*} P<0.05$ and ${ }^{* *} P<0.01$, by 2 -way ANOVA (B and $\mathbf{F}$ ), 1-way ANOVA (C and $\mathbf{G})$, or log-rank test ( $\mathbf{D}$ and $\mathbf{H})$. Data represent the mean \pm SD.

ration of ATP5H expression in P3 cells markedly reduced the level of HIF- $1 \alpha$ as well as p-AKT and p-ERK (Supplemental Figure 10). Therefore, ATP5H loss, ROS gain, and HIF-1 $\alpha$ activation act in a linear pathway to govern the resistance of cancer cells to multiple classes of therapy.

We next explored the influence of HIF-1 $\alpha$ downstream of Atp5h or ATP5H silencing on the development of multimodal resistance to therapy after immune editing. Hifla or HIF1A knockdown reversed the AKT/ERK activation and VEGF secretion induced by Atp5h or ATP5H knockdown (Figure 7, G and $\mathrm{H}$ ), indicating that the effect of ATP5H loss on resistance is dependent on normoxic HIF-1 $\alpha$. Indeed, Hifla or HIF1A knockdown restored the susceptibility of Atp5h-or ATP5H-depleted tumor cells to immunotherapy, chemotherapy, and radiation (Figure 7I) and diminished the sphere-forming and invasive capacity of these tumor cells (Figure 7 , J and $\mathrm{K})$. Thus, we conclude that the normoxic HIF- $1 \alpha$ activation is a key control point of multimodal resistance to cancer therapy induced by the loss of ATP5H.

Loss of ATP synthase facilitates multimodal resistance following drug selection. We next investigated whether, as with immune editing, drug selection would also prompt loss of ATP synthase. To do this, we established cisplatin-refractory tumor cells, termed CaSki CR and H1299 CR, by in vivo or in vitro drug selection, respectively. We also used A2780 CP20 tumor cells, a cisplatin-refractory variant of $A 2780$ human ovarian cancer cells. We found that after drug selection, cisplatin-refractory tumor cells downregulated ATP5H, upregulated ROS and normoxic HIF- $1 \alpha$, and activated the
AKT/ERK signaling pathway (Figure 8, A and B, and Supplemental Figure 11A). Notably, after drug selection, the tumor cells also acquired multimodal resistance to immunotherapy, chemotherapy, and radiotherapy (Figure $8 \mathrm{C}$ ) and exhibited stem-like and invasive features (Figure 8D and Supplemental Figure 11A). Infusion of antioxidants decreased HIF- $1 \alpha$ signaling, reversed cross-resistance, and diminished the sphere-forming and invasive capacity of tumor cells (Figure 8, E-G and Supplemental Figure 11B). We treated NOD/SCID mice bearing refractory tumor cells with different combinations of cisplatin and hydrogel-encapsulated NAC (Figure $8 \mathrm{H}$ ). We observed that HIF-1 $\alpha$ levels were decreased by $90 \%$ in the tumor microenvironment of NAC-treated mice (Supplemental Figure 11C). While cisplatin alone had no effect on tumor growth, NAC delivery and cotreatment with cisplatin and NAC retarded tumor growth (Figure 8, I and J) and prolonged survival of the mice (Figure $8 \mathrm{~K}$ ). Thus, we conclude that, as is the case with immune editing, drug selection can incite multimodal resistance to therapy. Crucially, we found that this cross-resistance is due to mitochondrial metabolic reprogramming mediated by ATP synthase loss and can be reversed by the delivery of antioxidants.

The ATP5H/normoxic HIF-1 $\alpha$ metabolic reprogramming pathway is widely conserved in human cancer. To evaluate the role of the ATP5H/HIF- $1 \alpha$ axis in human cancer, we examined the expression of ATP5H and HIF-1 $\alpha$ proteins in a large panel of human tumor cells, including those derived from patients with cervical, colon, liver, breast, prostate, lung, ovarian, or skin cancer. In all of these cells, we observed an inverse relationship between ATP5H and 
A
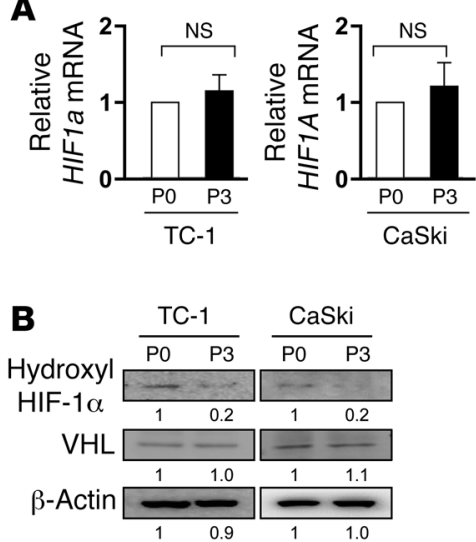

E

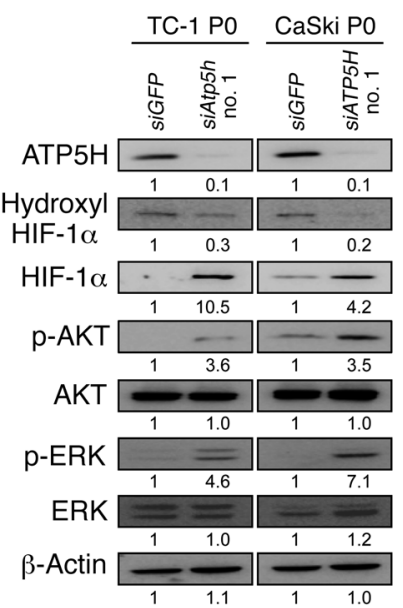

C

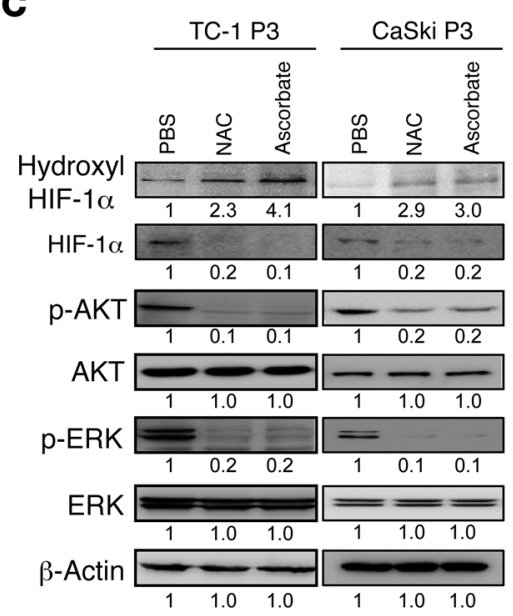

D

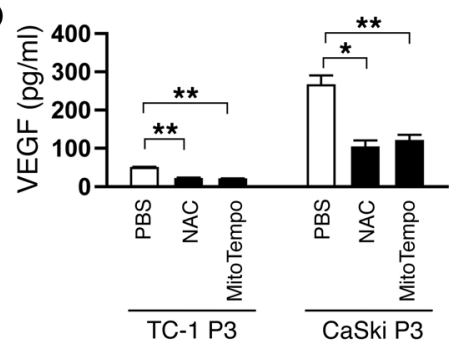

I

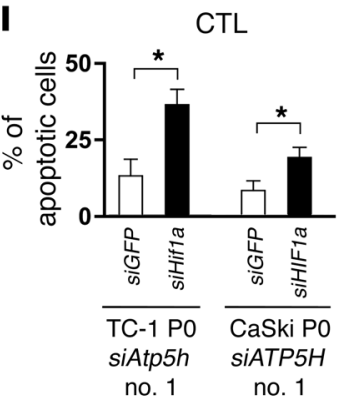

$\mathbf{F}$

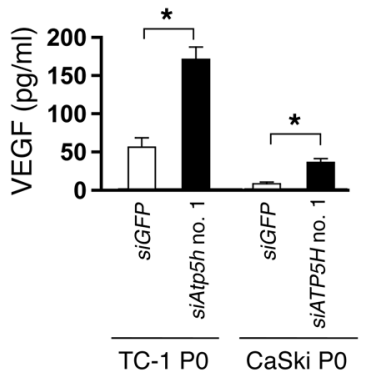

G

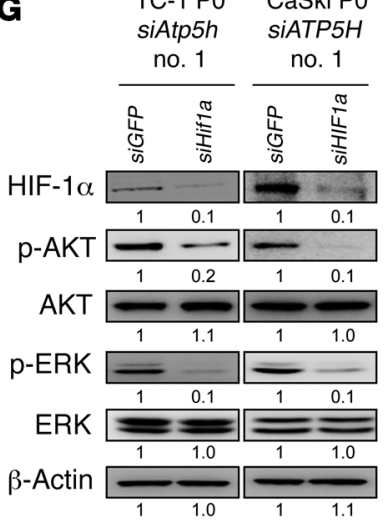

H

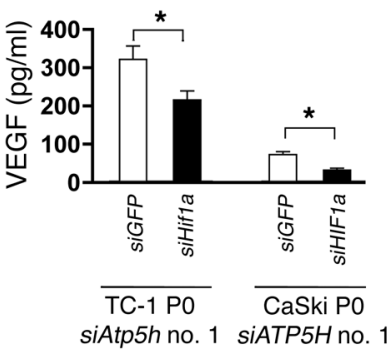

K
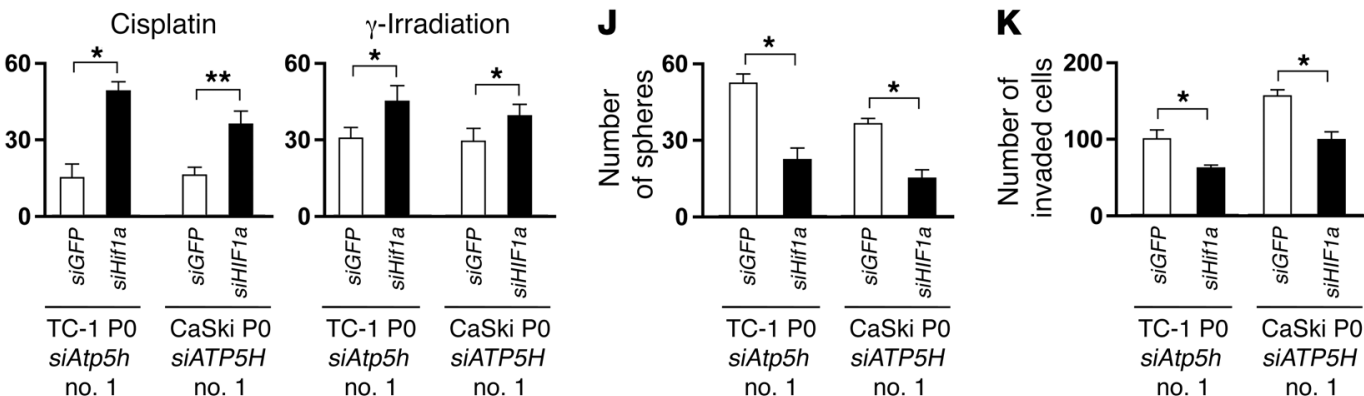

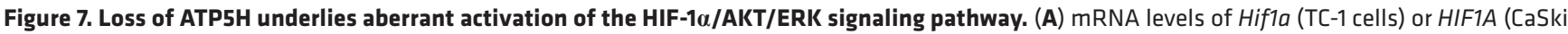
cells) in TC-1 or CaSki cells without (PO) or with (P3) immune editing was probed by qRT-PCR. (B) Hydroxylated HIF-1 $\alpha$ protein and VHL expression were probed by Western blotting (numbers below the blots are densitometric values). (C) Expression of HIF-1 $\alpha$ as well as phosphorylated and total AKT and ERK in TC-1 or CaSki P3 cells was determined by Western blotting in the absence or presence of antioxidants (i.e., NAC or ascorbate). Numbers below the blots are densitometric values. (D) VEGF levels in supernatant were determined by ELISA in TC-1 or CaSki P3 cells treated with or without antioxidants. (E and F) TC-1 or CaSki PO cells were transfected with the indicated siRNAs. (E) Western blot analysis of HIF-1 $\alpha$ as well as p-AKT and p-ERK levels in these cells. Numbers below the blots are densitometric values. (F) ELISA of VEGF secretion by these cells. (G-K) siAtp5h- or siATP5H-transfected TC-1 and CaSki cells were cotransfected with the indicated siRNAs. (C) HIF-1 $\alpha$ as well as p-AKT and p-ERK levels in these cells were probed by Western blotting (numbers below the blots are densitometric values). (H) VEGF levels in the supernatant of these cells were determined by ELISA. (I) Cells were exposed to E7-specific CTLs, cisplatin, or $\gamma$-irradiation. The frequency of apoptotic cells was determined by flow cytometric analysis of caspase-3 activation. The degree of stem-like (J) and invasive (K) phenotypes in these cells was determined by sphere-forming or Matrigel migration assay, respectively. All experiments were performed in triplicate under normoxic conditions. ${ }^{*} P<0.05$ and ${ }^{* *} P<0.01$, by 2 -tailed Student's $t$ test (A, $\mathbf{H}$, and $\mathbf{F}$-K) or 1 -way ANOVA (D). Data represent the mean \pm SD. 
HIF-1 $\alpha$ expression (Figure 9, A and B). Furthermore, knockdown of $A T P 5 H$ expression in these tumor cells markedly upregulated normoxic HIF-1 $\alpha$ and ROS (Figure 9, C and D, and Supplemental Figure 12). ATP5H knockdown also conferred multimodality resistance to immunotherapy, chemotherapy, and radiotherapy (Figure $9, \mathrm{E}-\mathrm{G}$ ), as well as a more pronounced sphere-forming, invasive phenotype (Figure 9, $\mathrm{H}$ and I). These results demonstrate that the biochemical and functional properties of the ATP5H/HIF-1 $\alpha$ axis are conserved across multiple types of cancer cells.

Loss of ATP synthase is associated with aggressive disease and poor prognosis in cancer patients. To determine the clinical relevance of the ATP5H/HIF-1 $\alpha$ axis in human cancer, we first performed IHC for ATP5H and HIF- $1 \alpha$ on tumor tissue sections from patients with established cervical carcinoma at various defined stages (Supplemental Figure 13). We found that patients with earlystage (I-IIA) cancer had high ATP5H and low HIF-1 $\alpha$ expression levels; by contrast, patients with late-stage (IIB-IV) cancer had low ATP5H and high HIF-1 $\alpha$ expression levels (Figure 10A). In addition, when we stratified patients into groups of those with an ATP5H ${ }^{\text {hi }}$ HIF- $1 \alpha^{\text {lo }}$ versus ATP5 $\mathrm{H}^{\text {lo }}$ HIF- $1 \alpha^{\text {hi }}$ tumor phenotype, we found, strikingly, that $80 \%$ of the patients with early-stage cancer had the ATP $5 \mathrm{H}^{\text {hi }}$ HIF- $1 \alpha^{\text {lo }}$ phenotype, while $90 \%$ of the patients with late-stage cancer had the ATP5 $\mathrm{H}^{\mathrm{lo}} \mathrm{HIF}-1^{\text {hi }}$ phenotype $(P<$ 0.001) (Figure 10B), reflecting a clinically compelling correlation between activation of the ATP5H/HIF- $1 \alpha$ axis and tumor aggressiveness. More important, patients with low ATP5H expression in the tumor also had a poor prognosis compared with those with high ATP5H expression $(P<0.002)$, and the effect of ATP5H loss became even more pronounced when examined in conjunction with HIF-1 $\alpha$ status $(P<0.001)$ (Figure 10C). In fact, over $80 \%$ of patients with the ATP5H $\mathrm{H}^{\text {lo }} \mathrm{HIF}-1 \alpha^{\text {hi }}$ tumor phenotype had a large tumor mass $(>4 \mathrm{~cm})$, lymph node metastasis, and poor response to chemoradiotherapy, consistent with our data from cell-based systems in this study (Table 1 and Supplemental Table 2). On the other hand, the majority of patients with the ATP5H $\mathrm{H}^{\text {hi }} \mathrm{HIF}$ $1 \alpha^{\text {lo }}$ tumor phenotype had a small tumor $(<4 \mathrm{~cm})$, no lymph node metastasis, and a positive response to chemoradiotherapy (Table 1 and Supplemental Table 2). Remarkably, ATP5H expression in the tumor was a stronger predictor of overall survival compared with disease stage/grade, tumor size, or presence of lymph node metastasis (Table 2). Thus, we conclude that the ATP5H/HIF-1 $\alpha$ axis is conserved in patients with cancer and serves as a clinical determinant of disease prognosis.

\section{Discussion}

The adaptation of tumor cells to host immune defenses has emerged as a major driving force for cancer onset and progression (38). This adaptation may occur through host-intrinsic events, such as the induction of tolerance to tumor antigen in tumorspecific CTLs, or the recruitment of Tregs or myeloid-derived suppressor cells to the tumor microenvironment (39). Alternatively, adaptation may be controlled by tumor-intrinsic events, such as loss of antigen or gain of resistance to apoptosis $(40,41)$. Here, we show that tumor adaptation to immune surveillance can be triggered by the metabolic state of tumor cells via mitochondrial reprogramming through loss of ATP synthase, in particular its $\mathrm{D}$ subunit (ATP5H). The link between host immune surveillance and tumor mitochondrial bioenergetics has not been previously explored. Furthermore, to our knowledge, we are the first to report a role for ATP5H in cancer immune escape.

It is intriguing, and perhaps counterintuitive, that ATP synthase loss would drive therapeutic resistance in cancer. Nonetheless, it has been well documented, in the phenomenon of the Warburg effect, that tumor cells rely chiefly on glycolysis, as opposed to mitochondrial oxidative phosphorylation, for ATP synthesis (42). In light of this, our results demonstrate that ATP5H loss in tumor cells after selection leads to increased glucose uptake and lactate production, suggesting that dysfunction of ATP synthesis contributes to the Warburg effect. We postulate that disruption of glycolysis in tumor cells (e.g., via inhibition of lactate dehydrogenase) may also restore susceptibility to therapy in refractory tumor cells.

Although loss of ATP synthase has been previously observed in cancer cells refractory to therapy, such as 5-FU-insensitive colon cancer cells (26), the underlying mechanisms responsible for linking ATP synthase loss to acquisition of resistance are largely unknown. Here, we have elucidated the signaling pathway by which resistance emerges downstream of ATP synthase loss. In this regard, we have found that expression of HIF-1 $\alpha$ is profoundly upregulated - under both normoxia and hypoxia - in tumor cells subjected to immune editing by tumor-specific CTLs compared with those without selection (28). While we have established that the HIF-1 $\alpha$ /VEGF axis drives immune escape (28), it is unclear how HIF-1 $\alpha$ protein expression is induced in tumor cells under immune editing. Our present study demonstrated that ATP5H loss-mediated mitochondrial dysfunction could accumulate ROS, which in turn stabilized HIF- $1 \alpha$ in normoxic conditions. The results indicated the crucial role of ATP5H loss as the origin of the gain of HIF- $1 \alpha$ in tumor cells.

Importantly, the inverse relationship between HIF-1 $\alpha$ and ATP5H expression was widely conserved in tumor cells derived from multiple types of human cancer cell lines, as well as in tumor tissue from patients with cervical cancer. Also, expression of ATP5H and HIF- $1 \alpha$ within the tumor correlated strongly with disease progression and survival in cancer patients. Thus, our data indicate that the expression status of ATP5H (either alone or in conjunction with HIF- $1 \alpha$ ) within tumor tissue may serve as an excellent prognostic marker. Furthermore, our results support the development of rational therapies aimed at restoring ATP synthase activity or reducing ROS accumulation. As proof of this principle, we demonstrated that treatment with antioxidants could abolish HIF-1 $\alpha$ expression in tumor cells following immune editing, restore sensitivity to lysis by CTLs, and effectively control tumor growth in mice transferred with tumor-specific CTLs. Therefore, the clinical administration of antioxidants may overcome the problem of acquired resistance to immune-based therapies.

Downregulation of ATP synthase subunits has been reported in multiple human carcinomas $(24,25)$. This raises the obvious question of precisely how ATP5H loss occurs in the course of cancer evolution. Our data point to epigenetic alteration at the Atp5h or $A T P 5 H$ gene locus as a result of immune editing. In particular, we have found that selection prompts histone deacetylation at the Atp5h or ATP5H promoter and provokes the transcriptional loss of ATP5H in tumor cells. It is important to define the chromatin modifier involved in this epigenetic process. Recently, we demon- 
A
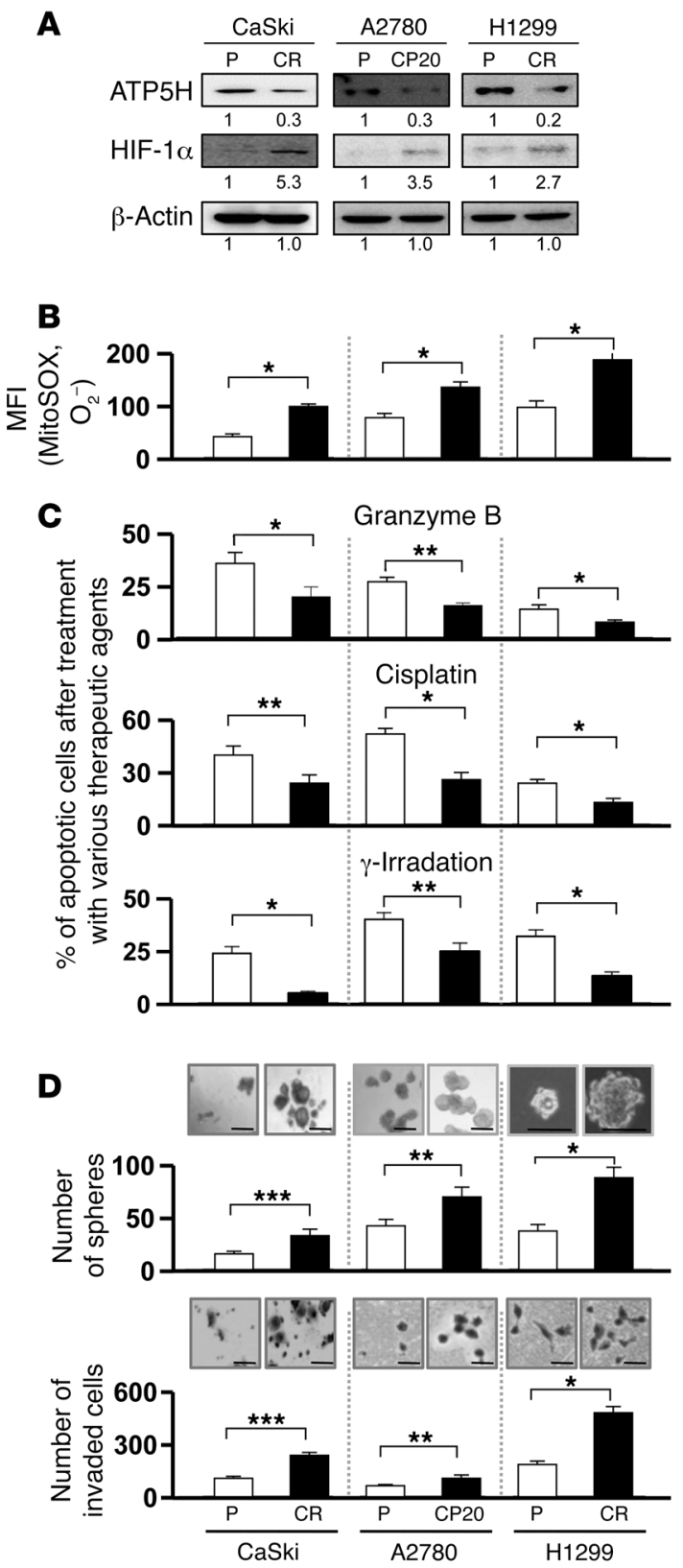

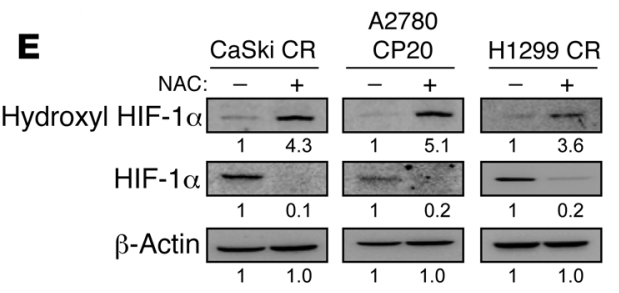

F

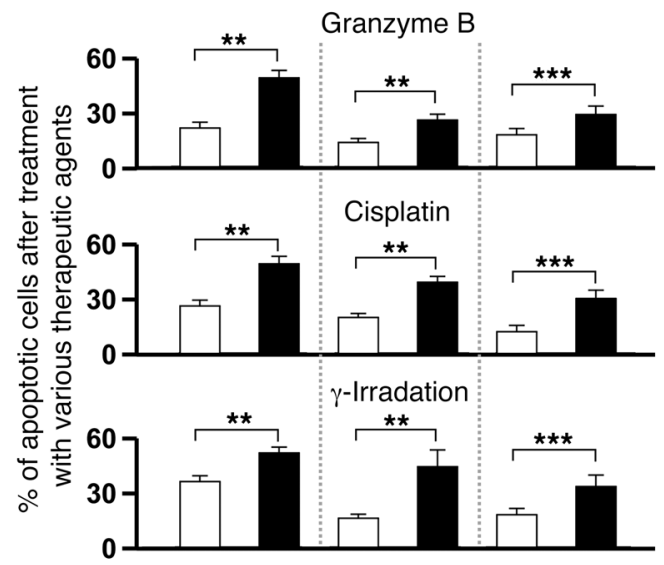

G

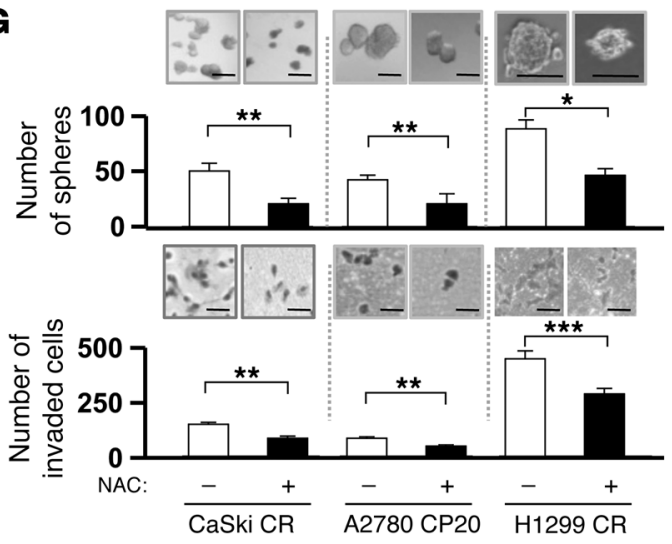

H

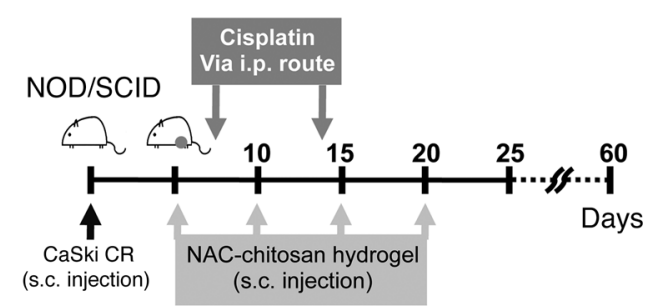

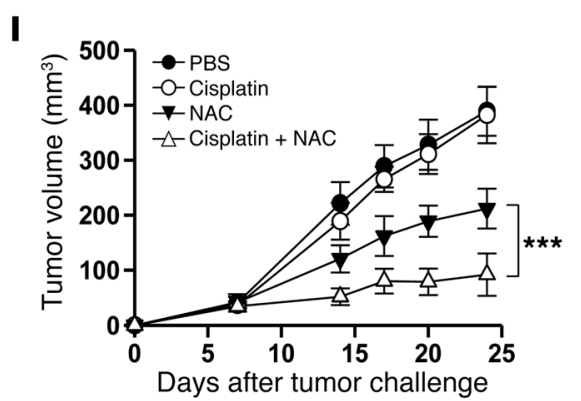
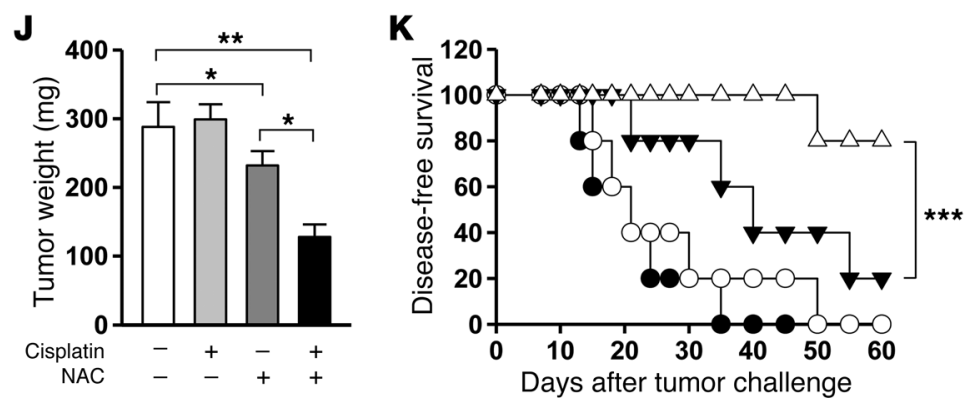
Figure 8. Chemoselection triggers ATP5H lost, mitochondrial reprogramming, and resistance to multimodal therapy. (A) ATP5H and HIF- $1 \alpha$ levels were probed in drug-sensitive $(P)$ or refractory (CR) human cancer cells by Western blotting (numbers below blots are densitometric values). Note: CP2O is a refractory version of A2780 cells. (B) ROS levels $\left(\mathrm{O}_{2}^{-}\right)$were determined by MitoSOX staining, followed by flow cytometric analysis. (C) $\mathrm{P}$ or CR cells were exposed to granzyme B, cisplatin, or $\gamma$-irradiation. The frequency of caspase-3-positive apoptotic cells was determined by flow cytometric analysis. (D) The degree of stem-like and invasive phenotypes of cells was determined by sphere-forming or Matrigel migration assay. Scale bars: $100 \mu \mathrm{m}$ (top) and $20 \mu \mathrm{m}$ (bottom), respectively. (E-G) CR tumor cells were incubated with or without antioxidants (NAC). (E) HIF-1 $\alpha$ levels were probed by Western blotting (numbers below blots are densitometric values). (F) Cells were exposed to granzyme B, cisplatin, or $\gamma$-irradiation. The frequency of caspase-3-positive apoptotic cells was determined by flow cytometric analysis. (G) The degree of stem-like and invasive phenotypes in cells was determined by sphere-forming or Matrigel migration assay. Scale bars: $100 \mu \mathrm{m}$ (top) and $20 \mu \mathrm{m}$ (bottom), respectively. (H) NOD/ SCID mice were inoculated with CaSki-CR cells. Mice were administered NAC $(0.1 \mathrm{mg} / \mathrm{kg})$ via chitosan hydrogel, together with cisplatin $(2 \mathrm{mg} / \mathrm{kg})$, at the indicated time points. (I) Tumor size was measured every 3 days. (J) The average tumor weight in each group was measured 21 days after tumor challenge. (K) Kaplan-Meier analysis of survival in each group. All in vitro experiments were performed in triplicate under normoxic conditions. For in vivo experiments, 10 mice from each group were used. ${ }^{*} P<0.05$, ${ }^{* *} P<$ 0.01 , and ${ }^{* * *} P<0.001$, by 2 -tailed Student's $t$ test (B-D, F, and $\left.\mathbf{G}\right)$, ANOVA (I and J), or log-rank test (K). Data represent the mean \pm SD.

strated the crucial role of HDAC1 in epigenetic silencing events of tumor cells during immune editing (27). Indeed, knockdown of $H d a c 1$ or HDAC1 in TC-1 and CaSki tumor cells after immune editing led to an increase in ATP5H levels (Figure 1, G and H), a reduction of ROS, and a decrease in HIF-1 $\alpha$ protein expression and VEGF secretion by tumor cells (Supplemental Figure 14). Therefore, our data indicate that HDAC1-mediated histone deacetylation facilitates epigenetic silencing of ATP5H in the course of immune editing and is hence a driver of cancer immune escape via mitochondrial reprogramming.

Surprisingly, our data also reveal defective ATP synthesis itself as the basis for resistance to therapy. Notably, when we ectopically silenced the expression of a different ATP synthase component (ATP5A) in tumor cells, we also observed a reduction in oxygen consumption, an elevation of ROS levels, as well as stabilization of HIF-1 $\alpha$ (Supplemental Figure 15). These data suggest that loss of other ATP synthase components (other than ATP5H) may similarly dampen the ATP synthase machinery and mitochondrial oxidative phosphorylation, leading to the development of multimodality resistance to therapy. Curiously, however, only the ATP5H subunit was consistently downregulated after immune selection, hinting that expression of the various components of the ATP synthase complex may be regulated in different ways.

It should be noted that the role of ROS in mediating tumor progression remains a complex and controversial matter. While elevated ROS levels in cancer cells have been suggested to contribute to cancer cell growth, angiogenesis, and metastasis (43), thus prompting the use of antioxidants as potential anticancer therapy, antioxidant supplementation has not been shown to confer a significant treatment advantage to cancer patients in various clinical trials (for review, see ref. 44). Furthermore, in certain tumor types, it has been suggested that upregulation of endogenous antioxidants by tumor cells helps facilitate progression and metastasis (45-47). In the current study, we observed that elevated ROS levels caused by treatment selection-induced ATP synthase dysfunction served as a critical process for the generation of multimodal therapy resistance and that antioxidant supplementation has the potential to restore the sensitivity of tumor cells to various treatments.

Altogether, our data provide evidence that selection pressure within the tumor microenvironment - such as that imposed by immunotherapy, chemotherapy, or radiotherapy - provokes the loss of ATP5H within a subset of tumor cells. These ATP5Hdeficient cells consequently undergo metabolic reprogramming and acquire a multimodal resistant phenotype. Furthermore, rational targeting of metabolic reprogramming within the tumor has the potential to overcome multimodal resistance to therapies.

\section{Methods}

Cells. The production and maintenance of HPV- $16 \mathrm{E} 7^{+} \mathrm{TC}-1$ cells (PO, $\mathrm{P} 1$, P2, P3) and CaSki cells (P0, P1, P2, P3) have been described previously $(18,28)$. To generate in vivo cisplatin-refractory CaSki cells, NOD/SCID mice were inoculated s.c. with CaSki cells. One week after the inoculation, CaSki cell-bearing mice were administered cisplatin $(2 \mathrm{mg} / \mathrm{kg}$, i.p.) once every three weeks. One of the outgrowth tumors resistant to cisplatin was explanted and expanded in vitro. After 2 rounds of in vivo drug selection, we obtained CaSki CR cells that were completely resistant to cisplatin in vivo. To generate in vitro H1299 CR cells, H1299 cells were treated with cisplatin $\left(\mathrm{IC}_{50}: 6.7 \mu \mathrm{M}\right)$ for 72 hours and then incubated for a further 72 hours without cisplatin. This sequential treatment was repeated for approximately 6 months. Ultimately, we obtained H1299 CR that was resistant to cisplatin in vitro. CUMC6, HeLa, CaSki, SiHa, DU145, PC-3, HepG2, HCT116, A549, H1299, NCI H1703, HCC95, SW620, LOVO, HT29, LNcap, MDA435, A2780, A2780 CP20, SK-OV3, OVCAR3, A375, and 526Mel cells were acquired from the American Type Culture Collection (ATCC); SNU-C4, SNU423, MCF-7, and MDA231 cells were acquired from the Korean Cell Line Bank (KCLB). All cell lines were recently authenticated.

Mice. Six- to eight-week-old female C57BL/6 and NOD/SCID mice were purchased from Central Lab Animal Inc.

Chemicals and reagents. NAC and ascorbate (MilliporeSigma) were used as ROS scavengers. DCF-DA and MitoSOX Red (Invitrogen, Thermo Fisher Scientific) were used for ROS detection in cells. MitoTempo (MilliporeSigma) was used as a specific scavenger of mitochondrial superoxide. Cisplatin and 5-FU (MilliporeSigma) were used as cytotoxic pharmacologic agents. 5-AzaC (MilliporeSigma) and TSA (Selleckchem) were used to inhibit DNA methyltransferase and HDAC, respectively. An ATP5H plasmid (RC207908, Origene Technologies) was used to restore ATP5H expression in treatment-resistant cells.

Antibodies. Primary antibodies against phosphorylated AKT (p-AKT) (no. 9271), AKT (no. 9272), p-ERK (no. 9101), ERK (no. 9102), HDAC1 (no. 5356), hydroxyl HIF-1 $\alpha$ (no. 3434), $\gamma$-H2AX (no. 2577), p-AMPK $\alpha$ (no. 2535), AMPK $\alpha$ (no. 2532), and LC3B (no. 2775) were obtained from Cell Signaling Technology. Primary antibodies against VHL (sc-5575, Santa Cruz Biotechnology), TRX (sc-20146, Santa Cruz Biotechnology), HIF-1 $\alpha$ (NB100-131, Novus Biologics), ATP5C (NBP215525, Novus Biologics), AcH3 (06-599, MilliporeSigma), AcH4 (06866, MilliporeSigma), MnSOD (06-984, MilliporeSigma), ATP5H (ab110275, Abcam), ATP5A (ab14748, Abcam), ATP5B (ab14730, Abcam), ATP5O (ab110276, Abcam), ATP5D (PA5-21361, Ther- 
A

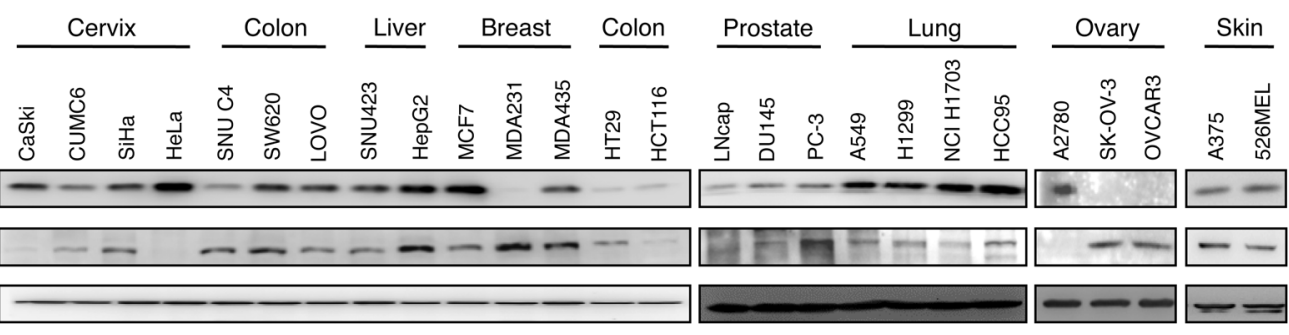

B

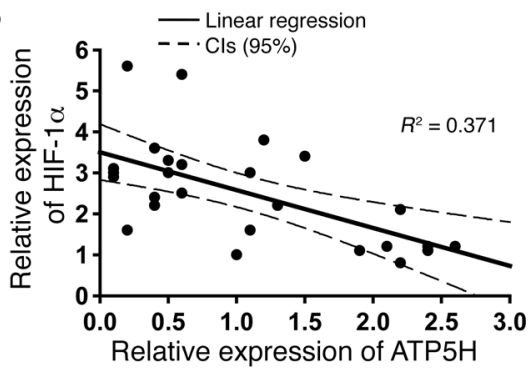

C

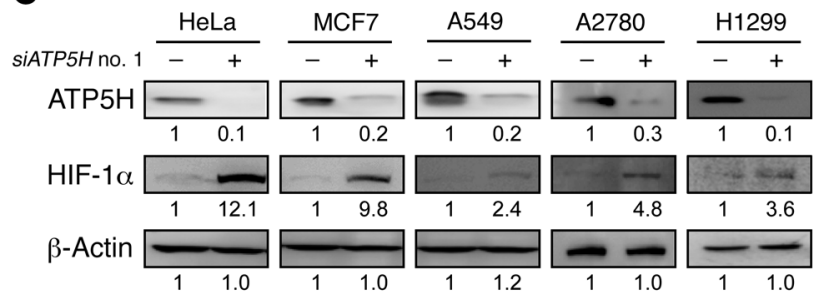

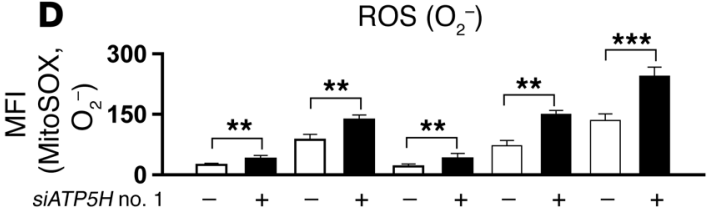

$\mathbf{F}$
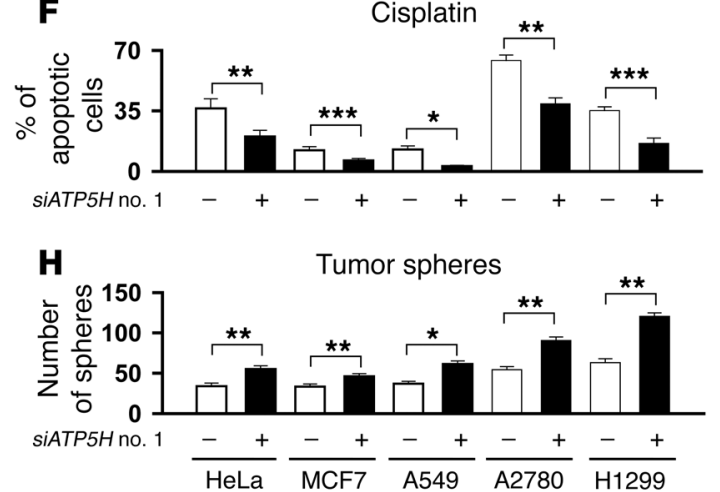

E

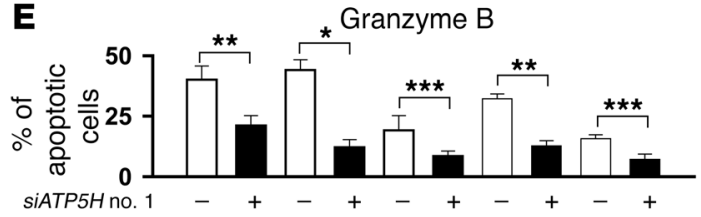

G
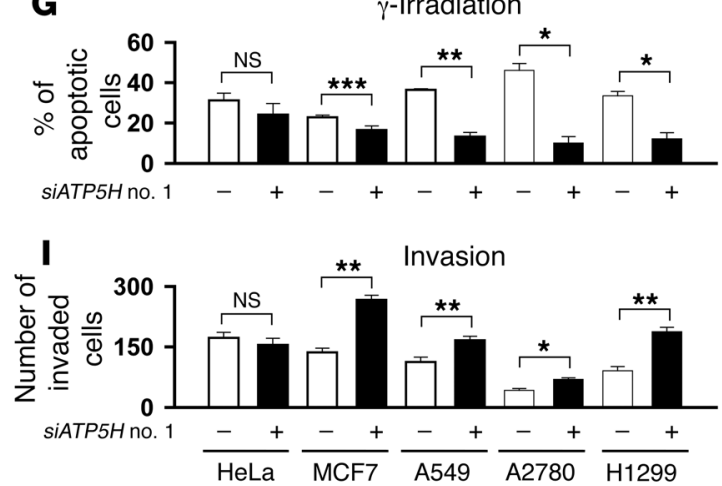

Figure 9. Broad-spectrum cross-resistance to conventional therapeutic agents mediated by ATP5H loss is widely conserved in human cancer. (A) Western blot analysis of HIF-1 $\alpha$ and ATP synthase subunit D (ATP5H) expression in various types of human tumor cells. (B) Linear regression analysis of the relationship between HIF-1 $\alpha$ and ATP5H expression in human cancers. Value 1 refers to the expression level of CaSki cells. (C-I) Various types of human tumor cells were transfected with siRNAs against GFP or ATP5H. (C) ATP5H and HIF-1 $\alpha$ levels were measured by Western blotting (numbers below the blots are densitometric values). (D) ROS $\left(\mathrm{O}_{2}^{-}\right)$abundance was determined by MitoSOX staining, followed by flow cytometric analysis. Transfected cells were exposed to granzyme $B(\mathbf{E})$, cisplatin $(\mathbf{F})$, or $\gamma$-irradiation $(\mathbf{C})$. The frequency of apoptotic cells was determined by flow cytometric analysis of caspase-3 activation. The degree of stem-like $(\mathbf{H})$ and invasive (I) phenotypes in transfected cells was determined by sphere-forming or Matrigel migration assay, respectively. All experiments were performed in triplicate under normoxic conditions. ${ }^{*} P<0.05,{ }^{* *} P<0.01$, and ${ }^{* * *} P<0.001$, by 2 -tailed Student's $t$ test. Data represent the mean $\pm \mathrm{SD}$.

mo Fisher Scientific), NANOG (A300-397A, Bethyl Laboratories), catalase (K90136S, BioDesign International), and $\beta$-actin (M177-3, MBL International) were acquired and used in this study.

siRNAs. Synthetic siRNAs were synthesized by Bineer (Deajeon, Korea). The synthetic siRNA sequences were 5'-CCAGATCTCGGCGAAGTAA-3' (sense) and 5'-UUACUUCGCCGAGAUCUGG-3' (antisense) for mouse Hifla siRNA and 5'-CCUAUAUCCCAAUGGAUGAUG-3' (sense) and 5'-CAUCAUCCAUUGGGAUAUAGG-3' (antisense) for human HIF1A siRNA. The sequences were 5'-AAUCUUCAGGGCAUUAUACUUCU-3' (sense) and 5'-AGAAGUCCCGAUAAUAUGAAGAAA-3' (antisense) for mouse siAtp5h (no. 1),
5'-UUAUGUUCCUCAUUUUCUCCA-3' (sense) and 5'-GAGAAAAUGAGGAACAUAAUU-3' (antisense) for mouse siAtp5h (no. 2), 5'-UAAUAAUUAUACAGUUAAA-3 (sense) and 5'-AUUAUUAAUAUGUCAAUUU-3' (antisense) for human siATP5H (no. 1), and 5'-GAAGCUCUGGCCCUUGUAU-3' (sense) and 5'-AUACAAGGGCCAGAGCUUC-3' (antisense) for human siATP5H (no. 2). The sequences were 5'-GAGUCAAAACAGAGGAUGA-3' (sense) and 5'-UCAUCCUCUGUUUUGACUC-3' (antisense) for mouse $\mathrm{Hdac1}$ and 5'-GAGUCAAAACAGAGGAUGA-3' (sense) and 5'-UCAUCCUCUGUUUUGACUC-3' (antisense) for human HDAC1. The siRNA for GFP (5'-GCAUCAAGGUGAACUUCAA-3' [sense]; 5'-UUGAAGUU- 
A
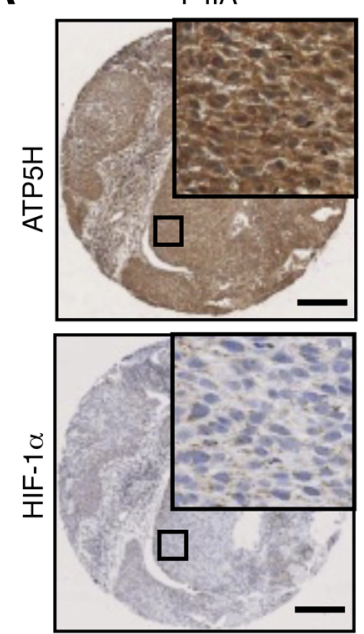

C

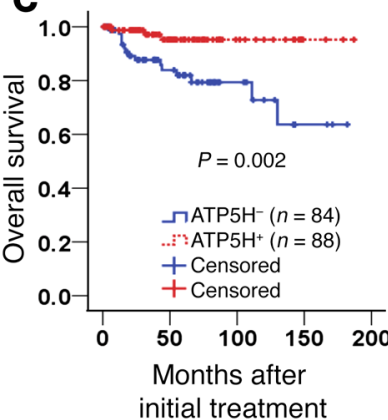

initial treatment
IIB-IV
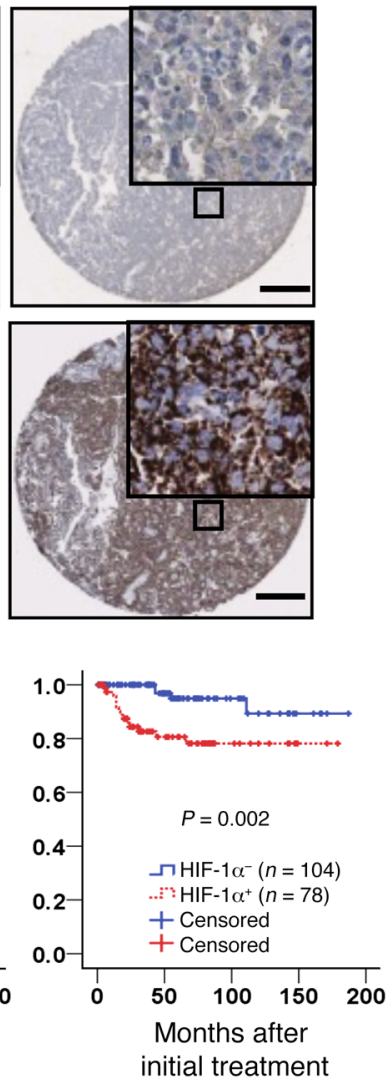

B

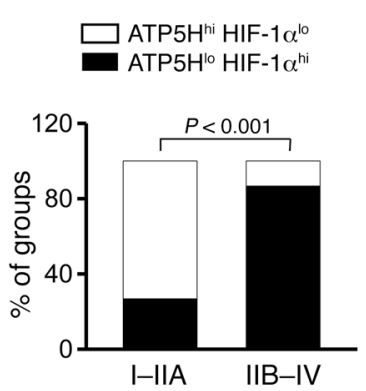

Figure 10. Expression status of ATP5H in tumor tissue influences disease outcome in patients with cancer. (A) Representative immunohistochemical analysis of ATP5H and HIF-1 $\alpha$ expression in tumor tissue from patients with cervical cancer. Scale bars: $200 \mu \mathrm{m}$. Original magnification, $\times 20$ (insets). The experiment was repeated at least 3 times. (B) Correlation between ATP5H and HIF-1 $\alpha$ expression in tumor tissue from patients with cervical cancer. (C) Kaplan-Meier analysis of disease-free survival among patients with cervical cancer, stratified by ATP5H and HIF-1 $\alpha$ expression status in tumor tissue specimens. Expression status was assessed by IHC and computed from an algorithm that considered both the intensity and total stained tissue area. $P$ values were calculated by 2 -tailed Student's $t$ test (B) or log-rank test (C).
CACCUUGAUGC- $3^{\prime}$ [antisense]) was used as a nonspecific control. Tumor cells $\left(10^{5}\right.$ cells) were transfected in 6-well plates with 100 pmol synthesized siRNAs with Lipofectamine 2000 (Invitrogen, Thermo Fisher Scientific).

Quantitative reverse transcription PCR. Total RNA was extracted with the RNeasy Mini Kit (QIAGEN) and treated with DNase (Ambion, Thermo Fisher Scientific). Quantitative reverse transcription PCR (qRT-PCR) mixtures were assembled with $1 \mu \mathrm{l}$ cDNA template, iQ SYBR Green Supermix (Bio-Rad), and primers for Hifla, HIF1A, Atp5h, or ATP5H. PCR was carried out for 40 cycles with the following thermal cycling conditions: $95^{\circ} \mathrm{C}$ for 10 seconds (denaturation) and $61^{\circ} \mathrm{C}$ for 60 seconds (annealing). All data were normalized to Actb mRNA expression levels.

Western blot analysis. Lysate extracted from a total of $1 \times 10^{5}$ cells was used to perform Western blot analysis, as described previously (18). Immunoreactive bands were developed with the Chemiluminescence ECL Detection System (Elpis-Biotech), and signals were detected using the LAS-4000 Mini Luminescent Image Analyzer (Fujifilm).

ChIP and quantitative ChIP assays. The ChIP Kit (MilliporeSigma) was used according to the manufacturer's instructions, and ChIP assays were performed as described previously (16). Briefly, cells $\left(10^{7}\right.$ per assay) were bathed in $1 \%$ formaldehyde (MilliporeSigma) at $25^{\circ} \mathrm{C}$ for 10 minutes for the crosslinking of proteins and DNA and then lysed in SDS buffer containing protease inhibitor. DNA was sheared to $0.2-$ to $1-\mathrm{kb}$ fragments by sonication using a Sonic Dismembrator Model 500 (Thermo Fisher Scientific). Immunoprecipitation was performed by incubation with $1 \mu \mathrm{g}$ anti-AcH3 and anti-AcH4 (MilliporeSigma) antibodies or rabbit IgG (MilliporeSigma) for 16 hours. To reverse protein-DNA crosslinking, the immunoprecipitated sample and input were incubated at $65^{\circ} \mathrm{C}$ overnight. After reversal of crosslinking, DNA fragments were purified on spin columns (MilliporeSigma). For qChIP assays, DNA immunoprecipitated by rabbit IgG, $\mathrm{AcH} 3$, or $\mathrm{AcH} 4$ was quantified by real-time qPCR using following the primer sets: $m A t p 5 h, 5^{\prime}$-GCCTTCCAGGACGGGACTCCAT-3' (forward) and 5'-GTCCGTGGAGATTCGACCCAACAC-3' (reverse) and hATP5H, 5'-TTGGCTGATCTGCGAAGCA-3' (forward) and 5'-GTAACGGAAGTGGGTCACGG-3' (reverse). Each sample was assayed in triplicate, and the amount of precipitated DNA was calculated as the percentage of input sample.

ELISA. VEGFA concentration in supernatant of TC-1 PO, P3, and CaSki PO and P3 cells collected at 48 hours was measured with a mouse and human VEGFA Quantikine ELISA Kit (R\&D Systems). The VEGFA concentration was normalized to the number of cells in each sample and depicted as picograms VEGFA per $10^{6}$ cells.

Confocal microscopy. TC-1 PO and P3 and CaSki PO and P3 cells were seeded at $2 \times 10^{4}$ cells per 2 -well culture slide (SPL Life Sciences) in DMEM supplemented with 10\% FBS and incubated overnight. To detect mitochondrial membrane potential, cells were treated with $5 \mu \mathrm{M}$ JC-1 dye (Invitrogen, Thermo Fisher Scientific) for 20 minutes. For detection of mitochondrial ROS, seeded cells were transfected with CellLight Mitochondria-GFP (Invitrogen, Thermo Fisher Scientific) and incubated overnight. Cells were treated with MitoSOX $(5 \mu \mathrm{M})$ (Invitrogen, Thermo Fisher Scientific) for 30 minutes. TC-1 PO and $\mathrm{P} 3$ and CaSki $\mathrm{PO}$ and $\mathrm{P} 3$ cells were fixed in $4 \%$ paraformaldehyde for 10 minutes. Cells were washed with PBS, treated with $0.2 \%$ Triton X-100, and blocked for 1 hour in $1 \%$ BSA. Nuclei were stained with DAPI (Invitrogen, Thermo Fisher Scientific). 


\section{Table 1. Correlation between the expression status of ATP5H and HIF-1a in the tumor microenvironment and tumor size, lymph node metastasis, and response to chemoradiotherapy in patients with cancer}

\begin{tabular}{|c|c|c|c|c|c|c|}
\hline \multirow{3}{*}{$\begin{array}{l}\text { Advanced tumor } \\
\text { phenotypes }\end{array}$} & \multicolumn{4}{|c|}{ Expression of combination marker ${ }^{A}$} & \multirow{3}{*}{$\begin{array}{c}\text { Correlation } \\
\text { coefficient }(R)\end{array}$} & \multirow[t]{3}{*}{$P$ value } \\
\hline & \multicolumn{2}{|c|}{ ATP5H-/HIF-1 $\alpha^{+}$} & \multicolumn{2}{|c|}{ ATP5H $\mathrm{H}^{+} / \mathrm{HIF}-1 \alpha^{-}$} & & \\
\hline & $\mathbf{N}$ & $\%$ & $\mathbf{N}$ & $\%$ & & \\
\hline Tumor size & & & & & -0.441 & $<0.001$ \\
\hline$\leq 4 \mathrm{~cm}$ & 18 & 40.9 & 26 & 59.1 & & \\
\hline$>4 \mathrm{~cm}$ & 20 & 87.0 & 3 & 13.0 & & \\
\hline LN metastasis & & & & & -0.403 & 0.002 \\
\hline Negative & 17 & 38.6 & 27 & 61.4 & & \\
\hline Positive & 12 & 85.7 & 2 & 14.3 & & \\
\hline Chemoradiation response & & & & & -0.400 & 0.012 \\
\hline Good & 11 & 36.7 & 19 & 63.3 & & \\
\hline Bad & 9 & 81.8 & 2 & 18.2 & & \\
\hline
\end{tabular}

${ }^{A}$ Cutoff value of ATP5H $\mathrm{H}^{+}$and HIF- $1 \alpha^{+}$is 6 or 8 points above the IHC score, respectively. FIGO, International Federation of Gynecology and Obstetrics; LN metastasis, lymph node metastasis; N, number.

peptide $(10 \mu \mathrm{g} / \mathrm{ml})$ for 1 hour and then mixed with cognate MART-1 or control noncognate NY-ESO1-specific $\mathrm{CD}^{+}$ CTLs at a 1:1 ratio and incubated for 4 hours at $37^{\circ} \mathrm{C}$. Cells were stained for active caspase- 3 as an index of apoptosis and examined by flow cytometry.

Granzyme B-mediated apoptosis assay. Recombinant human granzyme $\mathrm{B}$ (Enzo Life Sciences) was mixed with BioPorter Reagent (MilliporeSigma) at $25^{\circ} \mathrm{C}$ for 5 minutes. Tumor cells were mixed with BioPorter-granzyme B complexes for 4 hours at $37^{\circ} \mathrm{C}$. Cells were stained for active caspase- 3 as an index of apoptosis and examined by flow cytometry.

Cisplatin-mediated apoptosis assay. Tumor cells were seeded at $2 \times 10^{5}$ cells/well in 6-well plates 1 day before the assay. Cells were treated with 10

Mitochondria analysis. To detect mitochondrial membrane potential, cells were stained with $5 \mu \mathrm{M} \mathrm{JC}-1$ dye (Invitrogen, Thermo Fisher Scientific) for 20 minutes at $37^{\circ} \mathrm{C}$. The fluorescence intensity of JC- 1 aggregates was determined by flow cytometry as an index of the mitochondrial membrane potential. Flow cytometry was performed on a FACSCalibur device with CellQuest Pro software (both from BD Biosciences).

Metabolic analysis. ATP concentration was determined with an ATP Bioluminescent Assay Kit (MilliporeSigma). Cell lysate was incubated with ATP Assay Mix Solution containing luciferase and luciferin. Luciferase activity was measured as an index of ATP concentration with a TD-20/20 Luminometer (Turner Biosystems). The oxygen consumption rate (OCR) was measured at $37^{\circ} \mathrm{C}$ with an XF96 instrument (Seahorse Bioscience). Glucose uptake was analyzed using 2-deoxy-2-[(7-nitro-2,1,3-benzoxadiazol-4-yl) amino]-D-glucose (2-NBDG) (Invitrogen). Cells were cultured in glucose-free RPMI medium and then incubated with $50 \mu \mathrm{M} 2-\mathrm{NBDG}$ for 1 hour at $37^{\circ} \mathrm{C}$. 2-NBDG-positive cells were measured by flow cytometry. Lactate production was measured with a Lactate Assay Kit (BioVision). Cell culture medium was incubated with a lactate assay buffer containing lactate enzyme for 30 minutes. Lactate levels were measured with a uQuant Microplate Reader (BioTek) at 570nm wavelength.

Spontaneous apoptosis assay. TC-1 or CaSki PO cells were transfected with siGFP, siAtp5h (no. 1), siAtp5h (no. 2), siATP5H (no. 1), or siATP5H (no. 2). After 72 hours, the frequency of spontaneous apoptotic cells was determined by staining with anti-active caspase-3 antibody followed by flow cytometric analysis.

CTL-mediated apoptosis assay. TC- 1 cells or CaSki cells were labeled with CFSE (10 $\mu \mathrm{M})$ in DMEM supplemented with 0.1\% FBS. The CFSE-labeled TC-1 cells were mixed with E7-specific $\mathrm{CD}^{+}$CTLs at a 1:1 ratio and then incubated for 4 hours at $37^{\circ} \mathrm{C}$. The CFSElabeled CaSki cells were pulsed with MART1 cancer $\mu \mathrm{M}$ cisplatin for 24 hours and then harvested by trypsinization. The frequency of apoptotic cells was determined by staining with antiactive caspase- 3 antibody, followed by flow cytometric analysis.

$\gamma$-Irradiation-mediated viability assay. Cells were subjected to irradiation with a range of doses $(0,2,4,8$, or $10 \mathrm{~Gy})$ with a ${ }^{137} \mathrm{Cs}$ radiator at a rate of $2.01 \mathrm{~Gy} / \mathrm{min}$ and seeded onto tissue culture plates. After culture for 96 hours, viability was measured by trypan blue exclusion assay or active caspase- 3 assay. After treatment with NAC, the cells were irradiated at $2 \mathrm{~Gy}$.

Tumor sphere-forming assay. Cells were plated at $2 \times 10^{3}$ cells per well in 24-well, super-low-adherence vessels (Corning) containing serum-free DMEM-F12 (Thermo Fisher Scientific) supplemented with EGF (20 ng/ml), basic FGF (20 ng/ml), and 1× B27 supplement. Medium was replaced every 3 days to replenish nutrients. Cells were examined under a light microscope, and colonies of greater than $2 \mathrm{~mm}$ in diameter were counted as spheres.

In vitro invasion assay. Twenty-four-well culture inserts were precoated with Matrigel (Corning) Basement Membrane Matrix (BD Bio-

Table 2. Univariate and multivariate analyses of overall survival in patients with cervical

\begin{tabular}{|c|c|c|c|c|}
\hline \multirow[t]{2}{*}{ Variables } & \multicolumn{2}{|c|}{ Univariate analysis } & \multicolumn{2}{|c|}{ Multivariate analysis } \\
\hline & Hazard ratio $(95 \% \mathrm{Cl})$ & $P$ value & Hazard ratio $(95 \% \mathrm{Cl})$ & $P$ value \\
\hline Age (>50 yr) & $1.04(0.41-2.59)$ & 0.929 & NA & \\
\hline FIGO stage (>IIB) & 4.57 (1.81-11.51) & 0.001 & $3.55(1.20-10.50)$ & 0.022 \\
\hline Cell type (non-SCC) & $2.36(0.89-6.25)$ & 0.082 & NA & \\
\hline Grade (poor) & $2.27(0.89-5.77)$ & 0.083 & NA & \\
\hline Tumor size (>4 cm) & $1.71(0.66-4.40)$ & 0.263 & NA & \\
\hline LN metastasis & $2.39(0.79-7.25)$ & 0.123 & NA & \\
\hline $\mathrm{SCC} \mathrm{Ag}^{+}$ & $3.38(1.28-8.90)$ & 0.014 & $2.04(0.70-5.94)$ & 0.189 \\
\hline ATP5H ${ }^{-A}$ & 5.49 (1.59-18.99) & 0.007 & $3.99(1.06-14.94)$ & 0.040 \\
\hline HIF- $1 \alpha^{+B}$ & $4.87(1.60-14.82)$ & 0.005 & $3.59(1.14-11.29)$ & 0.029 \\
\hline ATP5H ${ }^{-} / \mathrm{HIF}-1 \alpha^{+}$ & $14.40(1.85-111.66)$ & 0.011 & 11.67 (1.35-100.72) & 0.025 \\
\hline
\end{tabular}

${ }^{A}$ Cutoff value of $\mathrm{ATP}^{+} \mathrm{H}^{+}$is 5 points above the IHC score. ${ }^{\mathrm{B} C u t o f f}$ of $\mathrm{HIF}-1 \alpha^{+}$is 6 points above the IHC score. NA, not applicable; SCC, squamous cell carcinoma. 
sciences) mixed with Opti-MEM (Thermo Fisher Scientific). Cells were suspended in serum-free medium, and $5 \times 10^{4}$ cells were added to the Transwell insert. Cells were incubated for 24 hours at $37^{\circ} \mathrm{C}$ in a $\mathrm{CO}_{2}$ incubator. Afterwards, cells that had migrated into the lower surface of the insert filter were washed twice with PBS, fixed with ethanol, and stained with $0.1 \%$ crystal violet dye. Cells were counted under a light microscope.

$\gamma$-H2AX analysis. Cells $\left(1 \times 10^{5}\right)$ were harvested by trypsinization, washed, and resuspended in PBS. To detect cellular $\gamma \mathrm{H} 2 \mathrm{AX}$ levels, cells were stained with anti- $\gamma-\mathrm{H} 2 \mathrm{AX}$ followed by Alexa Fluor 488 goat anti-rabbit IgG (A11034, Invitrogen, Thermo Fisher Scientific). The frequency of $\gamma \mathrm{H} 2 \mathrm{AX}$-positive cells was analyzed using a FACSVerse Flow Cytometer (BD Biosciences).

Tumor treatment experiments. C57BL/6 and NOD/SCID mice were inoculated s.c. with $10^{5}$ tumor cells. For adoptive transfer experiments, mice were administered $2 \times 10^{6} \mathrm{E} 7$-specific CTLs or an isotonic saline control by tail-vein injection 7 days after tumor challenge. For antioxidant combination therapy, mice were administered NAC $(100 \mathrm{mg} /$ $\mathrm{kg})$ in conjunction with adoptive $\mathrm{T}$ cell transfer or cisplatin $(2 \mathrm{mg} / \mathrm{kg})$ treatment. Tumor dimensions (length and width) were measured with a caliper every 3 to 4 days, and the tumor volume was calculated as length $(\mathrm{mm}) \times$ width $^{2}\left(\mathrm{~mm}^{2}\right) \times 0.52$.

$2 \mathrm{D}$ protein electrophoresis and MALDI-time-of-flight mass spectrometry. TC-1 P0 and TC-1 P3 cells in lysis buffer (7 M urea, $2 \mathrm{M}$ thiourea, 4.5\% CHAPS, $100 \mathrm{mM}$ DTE, $40 \mathrm{mM}$ Tris, $\mathrm{pH}$ 8.8) were applied to immobilized $\mathrm{pH}$ 3-10 nonlinear gradient strips (Amersham Biosciences). Isoelectric focusing on the first axis was performed at $80,000 \mathrm{Vh}$. Protein was run down a second orthogonal axis on a $9 \%$ to $16 \%$ linear gradient polyacrylamide gel at $40 \mathrm{~mA}$ for 5 hours. Protein was fixed in 40\% methanol and 5\% phosphoric acid for 1 hour, and the gel was stained with CBB G-250 for 12 hours. The gel was destained with $\mathrm{H}_{2} \mathrm{O}$ and scanned in a GS710 Densitometer (Bio-Rad). Twelve protein spots with differential intensities in PO versus P3 cells were excised and digested with endoproteinase. For MALDI-time-of-flight mass spectrometry (MALDI-TOF MS), peptide fragments derived from protein spots were concentrated on a POROS R2/Oligo R3 Column (Applied Biosystems). The column was first washed sequentially with $70 \%$ acetonitrile, $100 \%$ acetonitrile, and $50 \mathrm{mM}$ ammonium bicarbonate. Samples were applied to the column and eluted with cyano4-hydroxycinamic acid (MilliporeSigma). Samples were dissolved in $70 \%$ acetonitrile and $2 \%$ formic acid and loaded onto an Opti-TOF 384 Well MALDI Plate Insert (Applied Biosystems). MALDI-TOF MS was performed on a 4800 MALDI-TOF/TOF Analyzer (Applied Biosystems) equipped with a $355-\mathrm{nm} \mathrm{Nd:YAG} \mathrm{laser.} \mathrm{The} \mathrm{pressure} \mathrm{in} \mathrm{the}$ TOF analyzer is approximately $7.6 \times 10^{-7}$ Torr. The mass spectra were obtained in the reflectron mode with an accelerating voltage of $20 \mathrm{kV}$ and summed over 500 laser pulses. Calibration was performed using the 4700 Calibration Mixture (Applied Biosystems).

Tissue microarrays. Tissue microarrays were constructed with a TMArrayer machine (Pathology Devices), reviewed by a pathologist, and annotated on H\&E-stained slides. Four 1.0-mm-diameter cylindrical tissue cores consisting of a matched tumor specimen and normal epithelial tissue were then taken from corresponding regions of the donor paraffin blocks and transplanted into recipient paraffin blocks. Paraffin blocks were provided by the Korea Gynecologic Cancer Bank through the Bio and Medical Technology Development Program of the Ministry of Education, Science, and Technology of Korea.
IHC. Paraffin sections were cut to $5-\mu \mathrm{m}$ thickness, deparaffinized with xylene, and rehydrated with graded ethanol. Antigen retrieval was performed in a pressure cooker containing citrate buffer at $\mathrm{pH} 9.0$ (Dako) for ATP5H and pH 6.0 (Dako) for HIF-1 $\alpha$, and sections were incubated in $3 \% \mathrm{H}_{2} \mathrm{O}_{2}$ for 10 minutes. The sections were then incubated with anti-ATP5H antibody (mouse monoclonal, clone 7F9BG1, Invitrogen, Thermo Fisher Scientific) at 1:1,000 dilution for 2 hours or with anti-HIF-1 $\alpha$ antibody (mouse monoclonal, clone ESEE122, Novus Biologics) at 1:2,000 dilution for 30 minutes at room temperature. Samples were then incubated with an EnVision Dual Link (Dako) secondary antibody for 30 minutes. Samples were visualized with diaminobenzidine (Dako), counterstained with hematoxylin, dehydrated in ethanol, and cleared in xylene. Negative controls (substitution of the primary antibody with TBS) were run simultaneously.

Digital image analysis. IHC tissue sections were converted into digital format at $\times 20$ magnification with a Scanscope CS device (Aperio) and annotated with Digital Image Hub software (SlidePath). Annotated regions were subjected to image analysis with Tissue IA version 3.0 software (SlidePath). An algorithm was developed within the Tissue IA software program to quantify ATP5H and HIF-1 $\alpha$ expression on the basis of intensity and stained tissue area. These parameters were combined to assign an IHC score of 0 to 12 to each sample, representing minimal to maximal levels of expression, respectively.

Statistics. All data are representative of at least 3 separate experiments. Group differences were analyzed using a 2-tailed Student's $t$ test. Statistical analyses of multiple groups within experiments were performed using 1- or 2-way ANOVA. For IHC data, statistical analysis was performed using R software, version 3.1.2. The Mann-Whitney $U$ test was used to compare protein expression levels between groups. The $\chi^{2}$-test was used to assess associations between molecular markers. Survival distributions were estimated using the Kaplan-Meier method with the log-rank test. A Cox proportional hazards model was created to identify independent predictors of survival. In all cases, a $P$ value of less than 0.05 was considered statistically significant.

Study approval. All mice were maintained and handled in accordance with recommendations for the proper care and use of laboratory animals. The animal studies were conducted and performed under a protocol approved by the Korea University Institutional Animal Care and Use Committee (KUIACUC-2014-175). Approval for use of human material in research was obtained from the Korea Gynecologic Cancer Bank through the Bio and Medical Technology Development Program of the Ministry of Education, Science, and Technology (Seoul, South Korea).

\section{Author contributions}

KHS, YHL, HCB, CPM, AY, TCW, and TWK designed the study. KML and CY provided MART-1-specific CTLs. KHS, YHL, HCB, HJL, SRW, SJO, and YKB conducted the experiments. JHK, BWK, HC, EJC, JYC, SMH, TWC, and KTH performed ICH and data analysis. KHS, YHL, CPM, AY, TCW, and TWK wrote the manuscript. All authors read and approved the final manuscript.

\section{Acknowledgments}

We thank Emily Farmer for preparation of the manuscript and Lucy Wangaruro for administrative support (both from Johns Hopkins School of Medicine). This work was funded by the National Research Foundation of Korea (NRF-2017R1A2A1A17069818 and NRF-2013M3A9D3045881), the NIH Cervical Cancer Specialized 
Program of Research Excellence (SPORE) (P50 CA098252), the NIH Head and Neck Cancer SPORE (P50 CA96784-06), and NIH grant R01 CA114425-01.

Address correspondence to: T.C. Wu, Department of Pathology, Johns Hopkins School of Medicine, CRB II Room 309,
1550 Orleans St., Baltimore, Maryland 21231, USA. Phone: 410.614.3899; Email: wutc@jhmi.edu. Or to: Tae Woo Kim, Department of Biochemistry and Molecular Biology, College of Medicine, Korea University, 73, Inchon-ro, Seongbuk-gu, Seoul, 02841, South Korea. Phone: 82.2.2286.1301; Email: twkim0421@korea.com.
1. Gottesman MM. Mechanisms of cancer drug resistance. Annu Rev Med. 2002;53:615-627.

2. Holohan C, Van Schaeybroeck S, Longley DB, Johnston PG. Cancer drug resistance: an evolving paradigm. Nat Rev Cancer. 2013;13(10):714-726.

3. Letai AG. Diagnosing and exploiting cancer's addiction to blocks in apoptosis. Nat Rev Cancer. 2008;8(2):121-132.

4. Gottesman MM, Fojo T, Bates SE. Multidrug resistance in cancer: role of ATP-dependent transporters. Nat Rev Cancer. 2002;2(1):48-58.

5 . Bell DW, et al. Inherited susceptibility to lung cancer may be associated with the T790M drug resistance mutation in EGFR. Nat Genet. 2005;37(12):1315-1316.

6. Kobayashi S, et al. EGFR mutation and resistance of non-small-cell lung cancer to gefitinib. $N$ Engl J Med. 2005;352(8):786-792.

7. Longley DB, Harkin DP, Johnston PG. 5-fluorouracil: mechanisms of action and clinical strategies. Nat Rev Cancer. 2003;3(5):330-338.

8. Pao W, et al. Acquired resistance of lung adenocarcinomas to gefitinib or erlotinib is associated with a second mutation in the EGFR kinase domain. PLoS Med. 2005;2(3):e73.

9. Chapman $P B$, et al. Improved survival with vemurafenib in melanoma with BRAF V600E mutation. NEngl JMed. 2011;364(26):2507-2516.

10. Engelman JA, et al. MET amplification leads to gefitinib resistance in lung cancer by activating ERBB3 signaling. Science. 2007;316(5827):1039-1043.

11. Johannessen CM, et al. COT drives resistance to RAF inhibition through MAP kinase pathway reactivation. Nature. 2010;468(7326):968-972.

12. Nazarian R, et al. Melanomas acquire resistance to B-RAF(V600E) inhibition by RTK or N-RAS upregulation. Nature. 2010;468(7326):973-977.

13. Sergina NV, et al. Escape from HER-family tyrosine kinase inhibitor therapy by the kinaseinactive HER3. Nature. 2007;445(7126):437-441.

14. Villanueva J, et al. Acquired resistance to BRAF inhibitors mediated by a RAF kinase switch in melanoma can be overcome by cotargeting MEK and IGF-1R/PI3K. Cancer Cell. 2010;18(6):683-695.

15. Valent $P$, et al. Cancer stem cell definitions and terminology: the devil is in the details. Nat Rev Cancer. 2012;12(11):767-775.

16. Noh KH, et al. Nanog signaling in cancer promotes stem-like phenotype and immune evasion. J Clin Invest. 2012;122(11):4077-4093.

17. Mao CP, Wu T, Song KH, Kim TW. Immune- mediated tumor evolution: Nanog links the emergence of a stem like cancer cell state and immune evasion. Oncoimmunology. 2014;3(7):e947871.

18. Noh KH, et al. Activation of Akt as a mechanism for tumor immune evasion. Mol Ther. 2009;17(3):439-447.

19. Ferreira LM. Cancer metabolism: the Warburg effect today. Exp Mol Pathol. 2010;89(3):372-380.

20. Kaelin WG Jr, Thompson CB. Q\&A: Cancer: clues from cell metabolism. Nature. 2010;465(7298):562-564.

21. Hanahan D, Weinberg RA. Hallmarks of cancer: the next generation. Cell. 2011;144(5):646-674.

22. Biswas G, Anandatheerthavarada HK, Avadhani NG. Mechanism of mitochondrial stress-induced resistance to apoptosis in mitochondrial DNA-depleted C2C12 myocytes. Cell Death Differ. 2005;12(3):266-278.

23. Fulda S, Galluzzi L, Kroemer G. Targeting mitochondria for cancer therapy. Nat Rev Drug Discov. 2010;9(6):447-464.

24. Cuezva JM, et al. The bioenergetic signature of cancer: a marker of tumor progression. Cancer Res. 2002;62(22):6674-6681.

25. Isidoro A, et al. Alteration of the bioenergetic phenotype of mitochondria is a hallmark of breast, gastric, lung and oesophageal cancer. Biochem J. 2004;378(Pt 1):17-20.

26. Shin YK, et al. Down-regulation of mitochondrial F1F0-ATP synthase in human colon cancer cells with induced 5-fluorouracil resistance. Cancer Res. 2005;65(8):3162-3170.

27. Song KH, et al. HDAC1 Upregulation by NANOG promotes multidrug resistance and a stem-like phenotype in immune edited tumor cells. Cancer Res. 2017;77(18):5039-5053.

28. Lee YH, et al. Gain of HIF-1 $\alpha$ under normoxia in cancer mediates immune adaptation through the AKT/ERK and VEGFA axes. Clin Cancer Res. 2015;21(6):1438-1446.

29. Lee HJ, et al. NANOG signaling promotes metastatic capability of immunoedited tumor cells. Clin Exp Metastasis. 2015;32(5):429-439.

30. Korshunov SS, Skulachev VP, Starkov AA. High protonic potential actuates a mechanism of production of reactive oxygen species in mitochondria. FEBS Lett. 1997;416(1):15-18.

31. Turrens JF. Mitochondrial formation of reactive oxygen species. J Physiol.2003;552(pt 2):335-344.

32. Okon IS, Zou MH. Mitochondrial ROS and cancer drug resistance: Implications for therapy. Phar- macol Res. 2015;100:170-174.

33. Maxwell PH, et al. The tumour suppressor protein VHL targets hypoxia-inducible factors for oxygen-dependent proteolysis. Nature. 1999;399(6733):271-275.

34. Ivan M, et al. HIFalpha targeted for VHLmediated destruction by proline hydroxylation: implications for $\mathrm{O} 2$ sensing. Science. 2001;292(5516):464-468.

35. Jaakkola P, et al. Targeting of HIF- $\alpha$ to the von Hippel-Lindau ubiquitylation complex by O2-regulated prolyl hydroxylation. Science. 2001;292(5516):468-472.

36. Brunelle JK, et al. Oxygen sensing requires mitochondrial ROS but not oxidative phosphorylation. Cell Metab. 2005;1(6):409-414.

37. Mansfield KD, et al. Mitochondrial dysfunction resulting from loss of cytochrome c impairs cellular oxygen sensing and hypoxic HIF-alpha activation. Cell Metab. 2005;1(6):393-399.

38. Dunn GP, Old LJ, Schreiber RD. The three Es of cancer immunoediting. Annu Rev Immunol. 2004;22:329-360.

39. Vesely MD, Kershaw MH, Schreiber RD, Smyth MJ. Natural innate and adaptive immunity to cancer. Annu Rev Immunol. 2011;29:235-271.

40. DuPage M, Mazumdar C, Schmidt LM, Cheung AF, Jacks T. Expression of tumour-specific antigens underlies cancer immunoediting. Nature. 2012;482(7385):405-409.

41. Igney FH, Krammer PH. Immune escape of tumors: apoptosis resistance and tumor counterattack. JLeukoc Biol. 2002;71(6):907-920.

42. Vander Heiden MG, Cantley LC, Thompson CB. Understanding the Warburg effect: the metabolic requirements of cell proliferation. Science. 2009;324(5930):1029-1033.

43. Liou GY, Storz P. Reactive oxygen species in cancer. Free Radic Res. 2010;44(5):479-496.

44. Tong L, Chuang CC, Wu S, Zuo L. Reactive oxygen species in redox cancer therapy. Cancer Lett. 2015;367(1):18-25.

45. Wang $\mathrm{H}$, et al. NRF2 activation by antioxidant antidiabetic agents accelerates tumor metastasis. Sci Transl Med. 2016;8(334):334ra51.

46. Piskounova E, et al. Oxidative stress inhibits distant metastasis by human melanoma cells. Nature. 2015;527(7577):186-191.

47. DeNicola GM, et al. Oncogene-induced Nrf2 transcription promotes ROS detoxification and tumorigenesis. Nature. 2011;475(7354):106-109. 